\title{
The Integrated Analysis of Transcriptomics and Metabolomics Unveils the Therapeutical Effect of Asiatic Acid on Alcoholic Hepatitis in Rats
}

\section{Siyun Chen}

Guangxi Medical University

Yushen Huang

Guangxi Medical University

Hongmei Su

Guangxi Medical University

Wuchang Zhu

Guangxi Medical University

\section{Yuanyuan Wei}

Guangxi Medical University

\section{Yan Long}

Guangxi Medical University

Yanxia Shi

Guangxi Medical University

Jinbin Wei ( $\nabla$ wjbguangxi@sina.cn )

Guangxi Medical University https://orcid.org/0000-0002-6813-6428

\section{Research Article}

Keywords: Asiatic acid, Alcoholic hepatitis, NF-KB pathway, Keap1/Nrf2 pathway, Glycerophospholipid metabolism pathway

Posted Date: December 6th, 2021

DOl: https://doi.org/10.21203/rs.3.rs-1079343/v1

License: (c) (i) This work is licensed under a Creative Commons Attribution 4.0 International License. Read Full License

Version of Record: A version of this preprint was published at Inflammation on March 29th, 2022. See the published version at https://doi.org/10.1007/s10753-022-01660-x. 


\section{Abstract}

The present study was to investigate the therapeutical effects and mechanisms of Asiatic acid from Potentilla Chinensis against alcoholic hepatitis. Rats were intragastrically fed with alcohol for 12 weeks to induce alcoholic hepatitis and then treated with various drugs for further 12 weeks. The results showed that Asiatic acid significantly alleviated liver injury caused by alcohol in rats, as evidenced by the improved histological changes and the lower levels of AST, ALT, and TBIL. Besides, Asiatic acid significantly enhanced the activity of ADH and ALDH, promoting alcohol metabolism. Asiatic acid suppressed CYP2E1 activity and NADP ${ }^{+} / \mathrm{NADPH}$ ratio, resulting in low ROS production. Further study revealed that Asiatic acid markedly reduced hepatocyte apoptosis by regulating the expression levels of the caspase and Bcl-2 families. Moreover, Asiatic acid could regulate the Keap1/Nrf2 and NF-KB signaling pathway, attenuating oxidative stress and inflammation as a result. Interestingly, the comprehensive analysis of transcriptomics and metabolomics indicated that Asiatic acid regulated the gene expression of Gpat4 and thereby affected the biosynthesis of the metabolites (1-acyl-Sn-glycerol-3-phosphocholine, phosphatidylcholine, phosphatidylethanolamine, and phosphatidylserine), regulating the glycerophospholipid metabolism pathway and ultimately ameliorating hepatocyte damage. In conclusion, this study demonstrates that Asiatic acid can ameliorate alcoholic hepatitis by modulating the NF-KB and Keap1/Nrf2 signaling pathways and the glycerophospholipid metabolism pathway, which may be developed as a potential medicine for the treatment of alcoholic hepatitis.

\section{Introduction}

The liver is an important organ for drug metabolism and detoxification. Long-term overconsumption of alcohol may lead to the development of various alcohol-related liver diseases, including alcoholic fatty liver, alcoholic hepatitis, alcohol-related fibrosis, and cirrhosis[1]. Among alcoholic liver diseases, alcoholic hepatitis (AH) has 10-35\% morbidity and few therapeutic options[2]. AH is characterized by jaundice and progressive inflammatory liver injury, closely associated with multiple metabolic alterations [3]. Chronic alcohol exposure can activate alcohol dehydrogenase and aldehyde dehydrogenase, resulting in overproduction of acetate and upregulation of cytochrome P450 2E1 (CYP2E1) expression, which induces alcoholic oxidative stress, pro-inflammatory mediators release, and fatty acid oxidation [4]. Therefore, it is urgent to explore an effective therapy for $\mathrm{AH}$.

Inflammatory cytokine production is mainly regulated by Nuclear factor-kB (NF-KB) activation, which subsequently promotes the transcription of numerous inflammation-associated genes, including IL-1 $\beta$, IL- 6 , and TNF- $\alpha$, and provides the basis for the generation of these cytokines[5]. In addition, the oxidative stress induced by reactive oxygen species (ROS) plays an important role in the regulation of alcoholic hepatitis. A large amount of acetaldehyde and ROS are produced in the process of alcohol metabolism, and the excessive accumulation of ROS may trigger the oxidative stress response of the body[6]. Furthermore, many genes related to oxidative stress are regulated by the nuclear factor erythroid-2-related factor (Nrf2)[7], indicating that the NF-KB and Nrf2 signaling pathways may be the potential pathway to regulate inflammatory response and oxidative stress in alcoholic hepatitis.

In the last decade, metabolomics research has provided a unique advantage in identifying potential biological metabolic differences by analyzing metabolic changes caused by active substances[8, 9]. Metabolomics studies provide a framework for drug research by directly analyzing the phenotypic changes of drugs acting on tissues and elucidating their mechanisms [10]. Furthermore, transcriptomics has played a huge role in uncovering potential targets of disease. Hence, RNA-seq sequencing was used to observe the changes of mRNA and genes, and metabolomics based on UPLC-Q/TOF MS was applied to investigate the protective effect of AA on alcoholic hepatitis.

In our previous study, we found that Asiatic acid (AA) extracted from Potentilla Chinensis had a strong antiinflammatory and inhibitory effect on lipid accumulation[11]. However, whether AA has protective effects on AH remains 
unclear so far. Therefore, we performed integrated analyses of metabolomic and transcriptomic to reveal the underlying mechanism of AA against hepatitis in the present study.

\section{Materials And Methods}

\section{Ethanol-induced $\mathrm{AH}$ model in rats}

In our previous study, AA (purity $>98.0 \%$ ) was isolated from Potentilla Chinensis[12]. Silibinin was purchased from Tianjin Tasly Shengte Pharmaceutical Co., Ltd. (Tianjin, China). A total of 60 Sprague Dawley (SD) male rats (Quality certificate number: SCXK Gui 2014-0002), weighing $160 \pm 20 \mathrm{~g}$ were purchased from the Experimental Animal Center of Guangxi Medical University (Guangxi, China). All the procedures of animal care and experiment were approved by the Animal Ethics Committee of Guangxi Medical University.

The experiment schedule was shown in Fig. 1. Briefly, after one week of adaptive feeding, all the rats were randomly

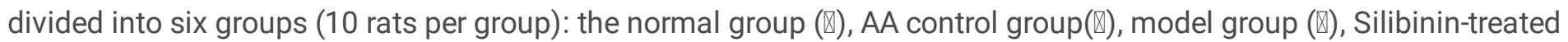
group ( () , low-dosages of $A A(\nabla)$ and high-dosages of $A A(\nabla)$ treatment groups. In this study, Silibinin, a natural medicine of liver protectors [13], was selected as the positive control. The alcoholic hepatitis was induced by intragastric administration of edible alcohol every day, and the therapeutic dosage of Silibinin and AA was chosen based on our previous study[11].

The ethanol dosages were given as follows: $30 \%$ ethanol $5.0 \mathrm{~g} / \mathrm{kg} / \mathrm{d}$ from 1 to 3 weeks, $35 \%$ ethanol $6.0 \mathrm{~g} / \mathrm{kg} / \mathrm{d}$ from 4 to 5 weeks, $40 \%$ ethanol $7.0 \mathrm{~g} / \mathrm{kg} / \mathrm{d}$ from 6 to 7 weeks, $40 \%$ ethanol $8.0 \mathrm{~g} / \mathrm{kg} / \mathrm{d}$ from 8 to 9 weeks, $40 \%$ ethanol 9.0 $\mathrm{g} / \mathrm{kg} / \mathrm{d}$ from 10 to 12 weeks, $40 \%$ ethanol $10.0 \mathrm{~g} / \mathrm{kg} / \mathrm{d}$ from 13 to 24 weeks. In addition to ethanol, rats in $\mathbb{V}, \mathrm{V}$, and $\mathrm{VI}$ groups were given $A A$; rats in the $\triangle$ group were given Silibinin from 13 to 24 weeks daily. At the modeling period, the body weights of all rats were weighed every two days.

At the end of the treatment, all the rats were anesthetized and sacrificed, the blood and liver tissue was stored in the $-80^{\circ}$ refrigerator for further examination.

\section{Histopathological and hepatocyte apoptosis examination}

A portion of liver tissues was prepared according to the previous studies[14]. The tissues were stained with hematoxylin and eosin (H\&E) staining and Masson's trichrome staining (Solarbio science\& technology, Beijing, China) for evaluating the pathological changes and hepatic collagen hyperplasia, respectively. Besides, the TUNEL staining (Beyotime Institute of Biotechnology, Shanghai, China) was used to observe the apoptosis hepatocyte.

\section{Biochemical analysis}

The blood of all rats was centrifuged, and then serum was collected. The serum activity of alanine aminotransferase (ALT), aspartate aminotransferase (AST), albumin (ALB), globulin (GLB), and total bilirubin (TBIL) was detected by an automatic biochemistry analyzer (Hitachi, Ltd., Kokubunji, Tokyo, Japan).

\section{Assay for alcohol metabolic enzyme activity}

The liver tissues were homogenized in normal saline and centrifuged at $2500 \times \mathrm{g}$ at $4^{\circ} \mathrm{C}$ for $10 \mathrm{~min}$. the supernatants were measured ADH and ALDH activity by commercially available diagnostic kits (Nanjing Jiancheng Bioengineering Institute, Nanjing, China), according to the manufacturer's instructions.

\section{Determination of oxidative stress and lipid peroxidation}


According to the manufacturer's instruction, the above supernatants were used to detect the activities of superoxide dismutase (SOD), Glutathione peroxidase (GSH-Px), Glutathione reductase (GSH-rd), malonaldehyde (MDA), and myeloperoxidase (MPO) using the commercially available kits (Nanjing Jiancheng Bioengineering Institute, Nanjing, Jiangsu, China).

\section{Determination of ROS, CYP2E1, and NADP ${ }^{+} / \mathrm{NADPH}$ ratio}

The fresh liver tissues were homogenized in PBS and centrifuged at $3000 \times \mathrm{g}$ at $4^{\circ} \mathrm{C}$ for $20 \mathrm{~min}$. The contents of reactive oxygen species (ROS) and cytochrome P450 2E1 (CYP2E1) in supernatants were detected with ELISA kits (Jiang\&Lai Biotechnology, shanghai, China) according to the manufacturer's instruction.

$\mathrm{NADP}^{+}$is the oxidized form of nicotinamide adenine dinucleotide phosphate (NADPH). NADP ${ }^{+}$NADPH is essential for maintaining cellular redox homeostasis and for modulating numerous biological events[15], playing a crucial role in alcohol metabolism [16]. The level of NADPH and NADP ${ }^{+}$were evaluated by the kit's specification (Nanjing Jiancheng Bioengineering Institute, Nanjing, Jiangsu, China).

\section{Western blot analysis}

Total protein from liver tissue was extracted with the protein extraction kits (Beyotime, Jiangsu, Nanjing, China); the nuclear and cytoplasmic proteins were isolated using nuclear extraction kits (Solarbio science\& technology, Beijing, China). According to the instruction manual, the liver was added to different reagents, homogenized, and centrifuged at $12,000 \mathrm{rpm}$ at $4^{\circ} \mathrm{C}$ for $20 \mathrm{~min}$, respectively. And then the concentration of proteins was detected with the Bicinchoninic acid (BCA) protein assay kit (Beyotime, Jiangsu, Nanjing, China). Finally, the protein samples were mixed with the loading buffer and boiled for $15 \mathrm{~min}$. Appropriate protein samples were isolated using 6\%-10\% SDS-polyacrylamide gel electrophoresis (PAGE), transferred onto PVDF membrane and blotted at $4^{\circ} \mathrm{C}$ overnight with different primary antibodies: Cleaved caspase 3, Cleaved caspase 8, Cleaved caspase 9, IKKa/ $\beta$, p-IKKa/ $\beta$, ІкBa, p-IKBa, NF-кB p65, p-NFKB p65, HO-1(1:1000, Cell Signaling Technology Inc.), Nrf2 and NQ01(1:1000, Abcam), as well as Bcl-2, Bax, Caspase 3, Caspase 8, Caspase 9, NF-KB p50, Keap1, Lamin B1 and GAPDH (1:1000 Proteintech). The PVDF membranes were incubated with the fluorescence-labeled goat anti-rabbit IgG (1:10,000 Licor) at room temperature under dark light for 1 hour. Subsequently, The PVDF was scanned with Odyssey infrared imaging system (Li-cor Bioscience, Lincoln, NE). Total protein and cytoplasmic protein were normalized to GAPDH, and nuclear proteins were normalized to Lamin B1.

\section{RT-PCR assay}

Total RNA was extracted from tissues by RNA Extraction Reagent Kit (Axygen Biotechnology, USA). The preparation and determination of the RT-PCR assay were carried out according to our previous study[17]. The PCR primers were synthesized by Sangon Biotech (Shanghai, China), GAPDH serving as a reference. The primers used in this experiment were listed in Table 1. 
Table 1

The sequences of primers used for RT-PCR.

\begin{tabular}{|c|c|c|}
\hline Genes & Forward primer $\left(5^{\prime}-3^{\prime}\right)$ & Reverse primer $\left(5^{\prime}-3^{\prime}\right)$ \\
\hline NF-кB p65 & АTСССтGстTССсСтTтстс & CTGTCTTATGGCTGAGGTCTGGT \\
\hline NF-кB p50 & GATGGGACGACACCTCTACACATA & CCCAAGAGTCGTCCAGGTCA \\
\hline TNF-a & GGCGTGTTCATCCGTTCTC & CTTCAGCGTCTCGTGTGTTTCT \\
\hline IL-6 & ATTGTATGAACAGCGATGATGCAC & CCAGGTAGAAACGGAACTCCAGA \\
\hline IL-1 $\beta$ & CTCACAGCAGCATCTCGACAAGAG & TCCACGGGCAAGACATAGGTAGC \\
\hline NQ01 & GGGGACATGAACGTCATTCTCT & AGTGGTGACTCCTCCCAGACAG \\
\hline $\mathrm{HO}-1$ & TGTCCCAGGATTTGTCCGAG & ACTGGGTTCTGCTTGTTTCGCT \\
\hline Nrf2 & TGCCCACATTCCCAAACAAG & TTGCTCCATGTCCTGCTGTA \\
\hline GAPDH & GGCACAGTCAAGGCTGAGAATG & ATGGTGGTGAAGACGCCAGTA \\
\hline
\end{tabular}

\section{Metabolome profiling}

The liver tissue was dissolved in the lysis buffer (formic acid: acetonitrile: water =10: 50: 40), and the supernatant was extracted and reserved. The metabolites were separated by ultra-high-performance liquid chromatography-tandem mass spectrometry (UPLC-Q/TOF MS, Waters, USA). The separation was achieved at $45^{\circ} \mathrm{C}$ on an Acquity UPLC BEH C18 column (2.1×100 mm, $1.7 \mathrm{~mm}$; Waters Corporation, Milford, MA). The mass spectra were acquired using a Xevo G2-XS Quadrupole Time-of-Flight mass spectrometer (Waters Corporation, Milford, USA) with Masslynx software v4.1 (Waters Corporation, Milford, USA). The mobile phase was composed of water and acetonitrile (containing $0.1 \%$ formic acid). The solvent gradient elution programs of samples were shown in Table 2. A $5 \mu$ l volume of sample was injected into the analytical column and the flow rate was $0.4 \mathrm{ml} / \mathrm{min}$.

Table 2

The solvent gradient elution programs of samples

\begin{tabular}{|llll|}
\hline Time (min) & Flow velocity $(\mathrm{mL} / \mathrm{min})$ & A\% & B\% \\
\hline 0 & 0.4 & 95 & 5 \\
\hline 1 & 0.4 & 80 & 20 \\
\hline 2.5 & 0.4 & 60 & 40 \\
\hline 9 & 0.4 & 0 & 100 \\
\hline 12 & 0.4 & 0 & 100 \\
12.5 & 0.4 & 95 & 5 \\
\hline 14.5 & 0.4 & 95 & 5 \\
\hline A: $0.1 \%$ Formic acid-aqueous solution B: 0.1 Formic acid-acetonitrile solution \\
\hline
\end{tabular}

To ensure the stability and accuracy of the data analysis, $10 \mu \mathrm{l}$ of supernatant from each sample was mixed as to quality control (QC) samples. Masslynx software v4.1 was used for identifying the original mass spectral data and normalizing the total ion intensity of each chromatogram. The raw data were analyzed by Progenesis QI and EZinfo 3.0 software. The potential metabolites were screened out based on the variable importance (VIP) in the projection values 
and Student's t-test. VIP $>1$ and $p<0.05$ were considered as statistically significant. Moreover, the HMDB IDs of metabolites were uploaded to the MetaboAnalyst database

(https://www.metaboanalyst.ca/MetaboAnalyst/ModuleView.xhtml) to identify the potential metabolic pathways.

\section{RNA-Seq profiling}

Total RNA was isolated from the livers using Trizol (Thermo Fisher Scientific, USA). The library was constructed with NEBNext UltraTM RNA Library Prep Kit (Illumina) and purified by Library beads (AMPure XP System). NovaSeq 6000 platform was used for sequencing. Reads were processed for quality by Trimmomatic and aligned to the rats' genome using the STAR alignment algorithm. The differentially expressed genes (DEGs) were identified by the algorithm of DESeq2 FDR analysis; $|\log 2 \mathrm{FC}|>1$ and $\mathrm{P}$-value $<0.05$ were considered a significant difference.

The volcano plot was drawn by the $\mathrm{R}$ language based on the differential gene analysis and the color was determined by the filtering criteria. DEGs were clustered into biological pathways by the DAVID database (https://david.ncifcrf.gov/). Furthermore, DEGs and the HMDB IDs of potential metabolites were submitted to the MetaboAnalyst database (https://www.metaboanalyst.ca/MetaboAnalyst/ModuleView.xhtml) to determine the metabolic pathways.

\section{Statistical analysis}

SPSS software Ver. 24. 0. (Chicago, IL, USA) was performed for statistical analysis. The data for each group were presented as means \pm SD and tested by one-way ANOVA with Turkey's test for post hoc multiple comparisons. In all cases, differences were considered statistically significant with a $p$-value $<0.05$.

\section{Results}

\section{AA treatment ameliorated alcohol-induced liver injury}

As shown in Fig. 2a, the liver/body weight ratio of the model group was the highest among all groups; the values of AA and Silibinin treatment groups were between the normal group and the model group. The indication of liver injury was evaluated by serum TBIL, ALB, GLB, ALT, and AST levels. Long-term consumption of alcohol led to a significantly elevated activity of AST, ALT, TBIL, as well as the ratio of ALB/GLB (Fig. 2b-d). However, Silibinin and AA treatment significantly reversed these abnormal changes.

To test the extent of liver tissue damage in rats, H\&E staining was examined. As shown in Fig. 2e, liver tissue of the normal control and AA groups showed an intact morphology, clear liver sinuses, without lipid droplets, and inflammatory infiltration. On the contrary, the model group revealed prominent liver damages, as evidenced by the form of cytoplasmic vacuolation, microvesicular steatosis, loss of cellular boundaries, and even large-scale inflammatory necrosis. However, Silibinin and AA therapy largely alleviated these liver damages. These results suggested that AA treatment significantly ameliorated alcohol-induced liver injury.

\section{AA attenuated alcohol-induced collagen accumulation}

The result of Masson's trichrome staining showed that an abundance of collagen deposition was observed in the model group compared to the normal control. While AA and Silibinin treatments significantly reduced collagen accumulation conditions (Fig. 2f). These results demonstrated that AA markedly inhibited the production and accumulation of collagen caused by chronic alcohol.

\section{AA treatment restored metabolic enzymes activity}

$\mathrm{ADH}$ and $\mathrm{ALDH}$ are important enzymes for alcohol metabolism. As indicated in Fig. 3a, the activity of ADH and ALDH was markedly decreased in the model group, while treatment with AA significantly increased the activity of these 
enzymes.

As shown in Fig. 3b-f, chronic alcohol administration caused an increased content of ROS and CYP2E1, as well as the ratio of $\mathrm{NADP}^{+} / \mathrm{NADPH}$. However, AA and Silibinin treatment significantly alleviated these phenomena. These data suggested that AA could alleviate the abnormal expression of metabolic enzymes caused by chronic alcohol administration.

\section{AA alleviated alcohol-induced hepatic oxidative stress}

Hepatic oxidative stress was evaluated by measuring myeloperoxidase, lipid peroxidation, and antioxidant function levels. As presented in Fig. $3 \mathrm{~g}$ and $\mathrm{h}$, chronic alcohol intake led to a significant increase in the myeloperoxidase level of MPO and the lipid peroxidation level of MDA. Meanwhile, the model group had the lowest antioxidant enzyme level of SOD, GSH-Px, and GSH-Rd (Fig. 3i). However, AA and Silibinin treatment strikingly decreased the contents of MPO and MDA and increased the activities of the antioxidant enzymes including SOD, GSH-Px, and GSH-Rd. These results indicated that AA inhibited chronic alcohol-induced oxidative stress, protecting the liver from damage.

\section{AA treatment suppressed pro-inflammatory cytokines}

Inflammation is one of the main pathogenic factors of alcoholic hepatitis. Compared to the normal, higher mRNA transcription levels of IL-6, IL-1 $\beta$, and TNF-a were observed in the model group. However, the abnormal levels of proinflammatory cytokines were significantly alleviated by AA and Silibinin treatment (Fig. 3j). These data indicated that AA markedly inhibited pro-inflammatory cytokines release.

\section{AA treatment alleviated alcohol-induced hepatocyte apoptosis}

To verify whether alcohol overconsumption affects the process of apoptosis and necrosis, hepatocyte apoptosis was evaluated by TUNEL staining in the present study. As shown in Fig. 4a, there were few TUNEL-positive hepatocytes in the normal group, while numerous TUNEL-positive hepatocytes were observed in the model group. Interestingly, AA and Silibinin treatments significantly reversed these abnormal changes.

Additionally, the apoptosis-related factors including Bcl-2, Bax, and caspases family were detected by Western blotting. As indicated in Fig. 4b-e, an oversupply of alcohol significantly increased the expression of pro-apoptotic protein Bax, cleaved caspase-3, cleaved caspase-8, and cleaved caspase-9, as well as reduced anti-apoptotic protein Bcl-2 expression. Treatment with AA significantly reversed these abnormal changes. These results suggested that $A A$ attenuated hepatocyte apoptosis by modulating the protein expression of the Bcl-2 and the caspases family.

\section{AA treatment activated the Nrf2 signaling pathway}

Because of the crucial role of the severe oxidative damage in the pathogenesis of alcoholic hepatitis, we further explored the protective mechanism of $\mathrm{AH}$ based on the Nrf2-mediated signaling pathway. Nrf2 is a transcription factor that regulates the antioxidant response, and the Keap1/Nrf2 pathway plays an important role in protecting the liver from damage[18]. As shown in Fig. 5a and b, AA and Silibinin treatments led to a significant increase in the levels of Nrf2 (nuclear), NQ01, and HO-1, while a remarkable decrease in the levels of Keap1 and Nrf2 (cytoplasm). Similarly, compared to the normal group, the transcription level of Nrf2, NQ01, and HO-1 mRNA in AA and Silibinin treatment groups were significantly up-regulated (Fig. $5 \mathrm{c}$ to e). Taken together, these data suggested that AA likely activated the Keap1/ Nrf2 signaling pathway, suppressing the oxidative reaction.

\section{AA treatment inhibited hepatic NF-kB activation}

Alcoholic hepatitis is a severe inflammatory disease, and NF-KB is a key transcriptional factor in regulating the expression of various pro-inflammatory mediators[19]. Therefore, to elucidate the underlying mechanism of AA against 
inflammatory response, the NF-кB pathway-related key proteins were further detected. As shown in Fig. 6, compared with the normal group, the protein expressions of NF-kB p50, p-NF-kB p65, p-IкBa, and p-IKKa/ $\beta$ were significantly increased in the model group. In addition, chronic alcohol abuse promoted NF-KB p65 transport from cytoplasm to the nucleus, and the transcription level of NF-KB p50 and NF-KB p65 mRNA was strikingly increased. While AA and Silibinin treatment reversed these abnormal expressions, indicating that $A A$ inhibited the inflammatory response via inhibiting the NF-kB pathway.

\section{Metabolome analysis}

As presented in Fig. 7a, the Principal Component Analysis (PCA) plot of QC samples showed that the points were tightly clustered together, and a similar trend was found in the analysis of Hotelling's T2 Range (Fig. 7b). These data indicated that the metabolomic analysis method with good repeatability and stability was suitable for the subsequent research.

As shown in Fig. 7c, each group formed a distinct cluster in primary component analysis (PCA). And the Volcano plot showed that a total of 1079 metabolites were differentially prominent expressed (Fig. 7d). The S-Plot and VIP diagram showed that there were 757 differential metabolites between the normal and the model group (Fig. 7e and f). The MetaboAnalyst analysis revealed that these differential metabolites were mainly enriched in the glycerophospholipid metabolism pathway (P-value $<0.05$ and Impact $>0.4$ ) (Fig. 7g), suggesting that the alcohol-induced AH model was likely to be associated with lipid metabolic disturbance.

Furthermore, 480 differential metabolites were identified between the AA and model groups (Fig. 7h and i), of which 16 metabolic pathways were obtained. Interestingly, the glycerophospholipid metabolism pathway was the most relevant one (P-value<0.05 and Impact >0.2) (Fig. 7j). Further analysis revealed that AA regulated the glycerophospholipid metabolism pathway likely by modulating the synthesis of LysoPC(20:1(11Z)) (LysoPC), PC

(16:1(9Z)/22:6(4Z,7Z,10Z,13Z,16Z,19Z)) (PC), PE (18:3(6Z,9Z,12Z)/22:6(4Z,7Z,10Z,13Z,16Z,19Z)) (PE), and PS (18:0/18:1(9Z)) (PS), ultimately ameliorating lipid metabolic disturbance (Fig. 7k).

\section{Transcriptomic analysis}

To explore the effect of AA on the global gene of alcoholic hepatitis in rats, transcriptome analysis was conducted in this study. As shown in Fig. 8a, the Principal Component Analysis (PCA) diagram showed a discrete distribution of genes between the model and AA groups. The Volcano and heatmap plot indicated that AA enhanced 96 genes expression and inhibited 107 genes expression (Fig. 8b and c). Meanwhile, the KEGG pathway analysis showed that long-term alcoholism might lead to a wide range of metabolic changes in rats because the metabolic pathway was the most relevant one (Fig. 8d and e). These data indicated that AA could ameliorate alcohol-induced metabolic disorders at a genetic level.

\section{Integrated analysis of transcriptomics and metabolomics}

To explore the systemic mechanism of AA on alcoholic hepatitis, an interaction network between DEGs and differential metabolites was analyzed by the MetaboAnalyst database. As shown in Fig. 9a and Table 3, multiple lipid-related pathways were obtained, such as Retinol metabolism, Glycerophospholipid metabolism, Taurine and hypotaurine metabolism, Linoleic acid metabolism, Steroid hormone biosynthesis ( $P$ value $<0.05$ ), and Biosynthesis of unsaturated fatty acids (Impact>0.8). These metabolic pathways may be the key metabolic pathway of AA in treating alcoholic hepatitis. Moreover, glycerophospholipid metabolism was the most relevant metabolic pathway that simultaneously included both the DEGs and differential metabolites. The further analysis revealed that the Gpat4 gene might affect the synthesis of four metabolites: LysoPC (1-acyl-Sn-glycerol-3-phosphocholine), PC (Phosphatidylcholine), PE (Phosphatidylethanolamine), and PS (Phosphatidylserine) (Fig. 9b). Besides, compared to the model group, AA greatly 
decreased the expression of LysoPC and increased the expression of PC, PS, and PE (Fig. 9c to f). These results suggested that AA might alleviate AH symptoms by regulating lipid metabolism, and Gpat4 may be a key gene in the alteration process.

Table 3

Analysis of metabolic pathway enrichment

\begin{tabular}{|c|c|c|c|c|c|c|}
\hline NO. & $\mathrm{m} / \mathrm{z}$ & RT (min) & Metabolites & HMDB ID & Trend ${ }^{1}$ & Related pathway \\
\hline 1 & 269.2268 & 8.89665 & 11-cis-Retinol & HMDB0006216 & $\downarrow^{*}$ & Retinol metabolism \\
\hline 2 & 828.4988 & 12.99195 & PS (18:0/18:1(9Z)) & HMDB0010163 & $\uparrow^{* *}$ & $\begin{array}{l}\text { Glycerophospholipid } \\
\text { metabolism }\end{array}$ \\
\hline 3 & 369.3506 & 9.560133 & 5beta-Cholestanone & HMDB0011182 & $\downarrow^{* \star}$ & $\begin{array}{l}\text { Taurine and } \\
\text { hypotaurine } \\
\text { metabolism }\end{array}$ \\
\hline 4 & 804.5529 & 9.377117 & $\begin{array}{l}\text { PC (16:1(9Z)/22:6 } \\
(4 Z, 7 Z, 10 Z, 13 Z, 16 Z, 19 Z))\end{array}$ & HMDB0008023 & $\uparrow * \star$ & $\begin{array}{l}\text { Linoleic acid } \\
\text { metabolism, } \\
\text { Arachidonic acid } \\
\text { metabolism } \\
\text { and } \\
\text { Glycerophospholipid } \\
\text { metabolism }\end{array}$ \\
\hline 5 & 784.4048 & 8.7651 & Aldosterone & HMDB0000037 & $\uparrow^{* *}$ & $\begin{array}{l}\text { Steroid hormone } \\
\text { biosynthesis }\end{array}$ \\
\hline 6 & 269.2268 & 8.89665 & 9-cis-Retinol & HMDB0006217 & $\downarrow^{*}$ & Retinol metabolism \\
\hline 7 & 768.4976 & 8.433367 & $\begin{array}{l}\text { PE }(18: 3(6 Z, 9 Z, 12 Z) / \\
22: 6(4 Z, 7 Z, 10 Z, 13 Z, 16 Z, 19 Z))\end{array}$ & HMDB0009144 & $\uparrow^{* *}$ & $\begin{array}{l}\text { Glycerophospholipid } \\
\text { metabolism }\end{array}$ \\
\hline 8 & 369.3506 & 9.560133 & Cholesterol & HMDB0000067 & $\downarrow^{\star *}$ & $\begin{array}{l}\text { Steroid hormone } \\
\text { biosynthesis }\end{array}$ \\
\hline 9 & 784.4048 & 8.7651 & Cortisone & HMDB0002802 & $\uparrow * \star$ & $\begin{array}{l}\text { Steroid hormone } \\
\text { biosynthesis }\end{array}$ \\
\hline 10 & 550.3862 & 8.050133 & LysoPC(20:1(11Z)) & HMDB0010391 & $\downarrow^{*}$ & $\begin{array}{l}\text { Glycerophospholipid } \\
\text { metabolism }\end{array}$ \\
\hline
\end{tabular}

\section{Discussion}

Alcoholic hepatitis is a disease characterized by hepatocyte de-differentiation and loss of hepatocyte regenerative capacity[20]. The ingredients from Chinese herbal have been proved to be a promising remedy for the treatment of liver injury [21]. In our previous studies, we have made a great effort to screen out the potential components of Potentilla Chinensis and found that AA may be a candidate drug for the treatment of alcoholic hepatitis. In this study, we tried to elucidate the underlying mechanism of AA against alcoholic hepatitis. The animal model of alcoholic hepatitis was induced by various concentrations of alcohol. We found that AA treatment could significantly attenuate liver damage, as evidenced by the H\&E staining with fewer microvesicular steatosis and inflammatory necrosis and the lower activity of ALT, AST, TBIL, and ALB/GLB. Also, Masson's trichrome staining showed that AA treatment could extremely decrease collagen accumulation. These data indicated that AA greatly inhibited alcohol-induced liver injury. 
Considering the important role of apoptosis on alcohol-induced liver injury, we decided to further observe cell apoptosis by TUNEL staining. On the other hand, the Bcl-2 family is an important apoptotic protein in the death receptor-mediated pathway, consisting of anti-apoptotic and pro-apoptotic proteins[22]. The caspase family is the main mediator that activates cell apoptosis; Caspase-3, caspase-8, and caspase- 9 are key nodes to control apoptosis in the regulatory network[23]. The present study showed that the model group presented a large number of cell apoptosis, while AA treatment significantly alleviated the condition. Besides, AA markedly inhibited the expression of the caspase family and the Bcl-2 family. Altogether, these data suggest that AA alleviated hepatocyte apoptosis by regulating the protein expression of the Bcl-2 family and caspases family.

To understand the potential mechanism of AA on hepatitis, alcohol metabolism and inflammatory damage were first considered. Ethanol oxidation mainly regulated by alcohol dehydrogenase (ADH) and acetaldehyde dehydrogenase (ALDH) leads to excessive ROS and oxidative stress, being the main contributors to alcoholic liver injury $[6,24,25]$. Besides, CYP2E1 is a subfamily of cytochrome P450, and its induction is related to ROS production[26]. Excessive ROS not only further leads to the imbalance of pro-oxidants and antioxidants, but also damages the lipid membrane, which eventually contributes to necrosis or apoptosis[27, 28]. Moreover, the proportion of ethanol is alternatively metabolized by the microsomal ethanol oxidizing system (MEOS) located within the smooth endoplasmic reticulum of hepatocytes, which consumes NADPH, generates $\mathrm{NADP}^{+}$, and dissipates heat to convert into acetaldehyde [29]. In the current study, we found that the contents of $A D H$ and ALDH were considerably enhanced, whereas the activity of ROS and the ratio of $\mathrm{NADP}^{+} / \mathrm{NADPH}$ were significantly decreased after AA treatment; Moreover, AA treatment significantly enhanced the activities of GSH-Rd, GSH-Px, and SOD, while decreased the levels of CYP2E1, MDA, and MPO. These results suggested that $\mathrm{AA}$ ameliorated alcohol toxicity by promoting alcohol metabolism, reducing oxidative stress, and restoring the antioxidative enzymes.

Generally, oxidative stress and inflammation were crucial inducements to cause alcoholic hepatic injury. In the present study, AA significantly inhibited the expression of the pro-inflammatory cytokines of IL-1 $\beta$, IL-6, and TNF- $a$, indicating that AA could effectively reduce the inflammatory infiltration caused by alcohol. Next, we focused on the underlying mechanism of AA on oxidative stress, and the Keap1/Nrf2 signaling pathway was investigated. Nrf2 is a transcription factor that regulates the expression of a series of antioxidant genes to maintain redox homeostasis in the liver, and Keap1 is a key regulator of antioxidant reaction[30]. Under normal physiological conditions, Keap1 stably binds to Nrf2 and immobilizes it in the cytoplasm[18]. When stimulated by inflammatory response or oxidative stress, Nrf2 separates from Keap1 and release into the nucleus [31-33]. The present study showed that the transcription levels of Nrf2 and downstream genes NQO1 and HO-1 mRNA significantly were increased after AA treatment. Similarly, AA significantly increased the expression of Nrf2 in the nucleus and significantly decreased the expression of Keap1 and Nrf2 (cytoplasm). The results suggest that AA could alleviate oxidative stress by activating the Keap1/Nrf2 signaling pathway, to some extent.

In addition to Keap1/Nrf2 signaling pathway, we also focused on the NF-kB signaling pathway. NF-kB transcription factor is a cell survival regulator, participated in cancer development by promoting the major inflammatory pathway activated during liver injury[34]. NF-kB is a protein family, which composed of RelA (p65), RelB, c-Rel, p50, and p52. In the resting state, NF-кBp65/p50 binds to IKBa to form a polymeric complex, which prevents them from entering the nucleus. The activation of $\mathrm{IKKa} / \beta$ can promote the phosphorylation and degradation of IKBa, which activates NF-kB and separates the polymer formed, releasing p65/p50 heterodimer to regulate the transcription of various genes[35]. In this study, the Western blot analysis showed that AA decreased the expression of NF-KB p50 and the phosphorylation of IKB-a, IKK- $\alpha / \beta, N F-k B$ p65. Besides, AA also promoted the transfer of NF-kB from the cytoplasm to the nucleus, suggesting that AA may inhibit the NF-KB signaling pathway expression, thus exerting an anti-inflammatory effect. 
Furthermore, to further understand the possible mechanisms of AA on alcoholic hepatitis as a whole, transcriptomics and metabolomics were performed in this study. The transcriptomic analysis revealed that there were 207 DEGs between the AA and model groups. Interestingly, the KEGG pathway analysis indicated that these genes were mainly involved in metabolic processes and several metabolic pathways.

According to the results of the transcriptomic analysis, UPLC-Q/TOF-MS was further used to analyze the effect of AA on liver metabolites. We found that AA alleviated liver damage, probably by regulating the glycerophospholipid metabolism pathway. To our knowledge, lipids are the essential metabolites, alterations in hepatic lipid homeostasis may account for the development of hepatic steatosis, which may progress to steatohepatitis, fibrosis, and cirrhosis[36]. There were reports that excess consumption of lysoPCs regulates a variety of biological processes, including cell proliferation and inflammation. Peroxisomes are dynamic organelles surrounded by a single membrane with a defined lipid composition, the major components of which are phosphatidylcholine (PC) and phosphatidylethanolamine (PE)[37]. Besides, PC and $\mathrm{PE}$ are important supporting nutrients for the liver, exert an obvious protective effect against liver damage from viruses, pharmaceuticals, alcoholism, and other toxic influences[36, 38]. Phosphatidylserine (PS) is a glycerol phospholipid with negatively charged head groups[39]. In this study, the analysis revealed 480 differential metabolites between the AA and model groups, which were largely enriched in the lipid-related metabolic pathways, and glycerophospholipid metabolism was the most relevant pathway. Using integrative analyses of transcriptomics and metabolomics, we found that $A A$ might affect the expression of the Gpat4 gene, thereby affecting the synthesis of the metabolites such as LysoPC (1acyl-Sn-glycerol-3-phosphocholine), PC (Phosphatidylcholine), PE (Phosphatidylethanolamine), and PS (Phosphatidylserine), and ultimately regulating the glycerophospholipid metabolism pathway. To sum up, AA significantly regulated gene profile and metabolic profile, protecting the liver from damage; the DEGs-mediated glycerophospholipid metabolism pathway might play an important role in the process.

\section{Conclusion}

AA significantly ameliorates alcohol-induced liver injury and inflammation, which is likely associated with several aspects (as shown in Fig. 10): (1) activating the Nrf2 signaling pathway to ameliorate oxidative stress; (2) inhibiting the NF-KB pathway to relieve inflammation; (3) attenuating hepatocyte damage by regulating the DEGs-mediated glycerophospholipid metabolism pathway. Our findings contribute to a comprehensive insight into the therapeutical effects of AA on liver damage, which might provide a potential remedy for treating alcoholic hepatitis in the future.

\section{Declarations}

\section{AUTHOR CONTRIBUTION}

This work was carried out with collaboration among all authors. Jinbin Wei designed the study and was in charge of the funds. Yushen Huang, Hongmei Su, Wuchang Zhu, and Yuanyuan Wei experimented. Yan Long and Yanxia Shi provided help to the experiment. Siyun Chen was responsible for statistical analysis and wrote the manuscript. All authors read and approved the final manuscript.

\section{FUNDING}

The authors gratefully acknowledged the financial support provided by the National Natural Science Foundation of China (No. 81660706), the Natural Science Foundation of Guangxi (Nos. 2018GXNSFAA050107), and the Scientific and Technological Innovation Major Base of Guangxi (No. 2018-15-Z04).

\section{AVAILABILITY OF DATA AND MATERIALS}


The raw data and materials about this article are available from the corresponding author.

\section{DECLARATIONS}

\section{Ethics Approval and Consent to Participate}

All experiments were carried out in strict accordance with the guidelines for the use of animal care in the laboratories of the Experimental Animal Center of Guangxi Medical University. All protocols were approved by an independent animal care and use committee.

\section{Consent for Publication}

Not applicable.

\section{Competing Interests}

The authors declare that there are no conflicts of interest.

\section{References}

1. Wan, Y., H. Wu, Y. Li, Z. Xu, J. Yang, C. Liu, Y. He, M. Wang, X. Wu, and Y. Zhang. 2020. viaTSG-6 Inhibits Oxidative Stress and Induces M2 Polarization of Hepatic Macrophages in Mice With Alcoholic Hepatitis Suppression of STAT3 Activation. Frontiers in pharmacology 11: 10.

2. Xiang, X., S. Hwang, D. Feng, V. Shah, and B. Gao: Interleukin-22 in alcoholic hepatitis and beyond. Hepatology international 2020, 14:667-676.

3. Takeuchi, M., P. Vidigal, M. Guerra, M. Hundt, M. Robert, M. Olave-Martinez, S. Aoki, T. Khamphaya, R. Kersten, and E. Kruglov, et al. 2021. Neutrophils interact with cholangiocytes to cause cholestatic changes in alcoholic hepatitis. Gut 70: 342-356.

4. Gala, K., and V. Vatsalya: Emerging Noninvasive Biomarkers, and Medical Management Strategies for Alcoholic Hepatitis: Present Understanding and Scope. Cells 2020, 9.

5. Bieghs, V., and C. Trautwein. 2013. The innate immune response during liver inflammation and metabolic disease. Trends in immunology 34: 446-452.

6. Han, Y., B. Glueck, D. Shapiro, A. Miller, S. Roychowdhury, and G. Cresci: Dietary Synbiotic Supplementation Protects Barrier Integrity of Hepatocytes and Liver Sinusoidal Endothelium in a Mouse Model of Chronic-Binge Ethanol Exposure. Nutrients. 2020. 12.

7. Li, Y., Y. Zeng, Q. Huang, S. Wen, Y. Wei, Y. Chen, X. Zhang, F. Bai, Z. Lu, J. Wei, and X. Lin. 2019. Helenalin from Centipeda minima ameliorates acute hepatic injury by protecting mitochondria function, activating Nrf2 pathway and inhibiting NF-KB activation. Biomedicine \& pharmacotherapy = Biomedecine \& pharmacotherapie 119: 109435.

8. Zheng, H., J. Wu, H. Huang, C. Meng, W. Li, T. Wei, and Z. Su. 2019. Metabolomics analysis of the protective effect of rubusoside on palmitic acid-induced lipotoxicity in INS-1 cells using UPLC-Q/TOF MS. Molecular omics 15: 222232.

9. Rachakonda, V., C. Gabbert, A. Raina, L. Bell, S. Cooper, S. Malik, and J. Behari. 2014. Serum metabolomic profiling in acute alcoholic hepatitis identifies multiple dysregulated pathways. PloS one 9: e113860.

10. Michelena, J., C. Alonso, I. Martínez-Arranz, J. Altamirano, R. Mayo, P. Sancho-Bru, R. Bataller, P. Ginès, A. Castro, and J. Caballería. 2019. Metabolomics Discloses a New Non-invasive Method for the Diagnosis and Prognosis of Patients with Alcoholic Hepatitis. Annals of hepatology 18: 144-154. 
11. Wang, D., L. Lao, X. Pang, Q. Qiao, L. Pang, Z. Feng, F. Bai, X. Sun, X. Lin, and J. Wei: Asiatic acid from Potentilla chinensis alleviates non-alcoholic fatty liver by regulating endoplasmic reticulum stress and lipid metabolism Int Immunopharmacol 2018, 65:256-267.

12. Wei, J., Q. Huang, R. Huang, Y. Chen, S. Lv, L. Wei, C. Liang, S. Liang, L. Zhuo, and X. Lin. 2013. Asiatic acid from Potentilla chinensis attenuate ethanol-induced hepatic injury via suppression of oxidative stress and Kupffer cell activation. Biological pharmaceutical bulletin 36: 1980-1989.

13. Federico, A., and M. Dallio, Loguercio: Silymarin/Silybin and Chronic Liver Disease: A Marriage of Many Years. Molecules 2017, 22.

14. Pang, X., Q. Qiao, S. Vonglorkham, Z. Feng, L. Pang, S. Chen, D. Wang, L. Lao, X. Lin, and J. Wei. 2020. Asiatic acid ameliorates acute hepatic injury by reducing endoplasmic reticulum stress and triggering hepatocyte autophagy. Biomed Pharmacother 129: 110375.

15. Xiao, W., R. S. Wang, D. E. Handy, and J. Loscalzo. 2018. NAD(H) and NADP(H) Redox Couples and Cellular Energy Metabolism. Antioxid Redox Signal 28: 251-272.

16. Piszczatowska, K., D. Przybylska, E. Sikora, and G. Mosieniak: Inhibition of NADPH Oxidases Activity by Diphenyleneiodonium Chloride as a Mechanism of Senescence Induction in Human Cancer Cells. Antioxidants 2020, 9.

17. Feng, Z., L. Pang, S. Chen, X. Pang, Y. Huang, Q. Qiao, Y. Wang, S. Vonglorkham, Q. Huang, X. Lin, and J. Wei. 2020. Didymin ameliorates dexamethasone-induced non-alcoholic fatty liver disease by inhibiting TLR4/NF-KB and PI3K/Akt pathways in C57BL/6J mice. Int Immunopharmaco/ 88: 107003.

18. Zhao, X., L. Gong, C. Wang, M. Liu, N. Hu, X. Dai, C. Peng, and Y. Li. 2021. Quercetin mitigates ethanol-induced hepatic steatosis in zebrafish via P2X7R-mediated PI3K/ Keap1/Nrf2 signaling pathway. Journal of ethnopharmacology 268: 113569.

19. Sehrawat, T., M. Liu, and V. Shah. 2020. The knowns and unknowns of treatment for alcoholic hepatitis. The lancet Gastroenterology hepatology international 5: 494-506.

20. Massey, V., A. Parrish, J. Argemi, M. Moreno, A. Mello, M. García-Rocha, J. Altamirano, G. Odena, L. Dubuquoy, and A. Louvet, et al. 2021. Integrated Multiomics Reveals Glucose Use Reprogramming and Identifies a Novel Hexokinase in Alcoholic Hepatitis. Gastroenterology 160: 1725-1740.e1722.

21. Gao, Y., L. X. Song, M. N. Jiang, G. Y. Ge, and Y. J. Jia. 2008. Effects of traditional chinese medicine on endotoxin and its receptors in rats with non-alcoholic steatohepatitis. Inflammation 31: 121-132.

22. Ginting, C., I. Lister, E. Girsang, W. Widowati, D. Yusepany, A. Azizah, and H. Kusuma. 2021. Piper crocatumHepatotoxicity prevention in Acetaminophen-induced HepG2 cells by red betel (Ruiz and Pav) extract from Indonesia via antioxidant, anti-inflammatory, and anti-necrotic. Heliyon 7: e05620.

23. Wei, X., H. Wang, X. Sun, X. Huang, W. Xu, Y. Liang, L. Liu, S. Mo, X. Lin, and J. Lin: 4-hydroxy-2(3H)-benzoxazolone alleviates acetaminophen-induced hepatic injury by inhibiting NF-KB and activating Nrf2/HO-1 signaling pathways. American journal of translational research. 2020. 12:2169-2180.

24. Sung, C. K., S. M. Kim, C. J. Oh, S. A. Yang, B. H. Han, and E. K. Mo. 2012. Taraxerone enhances alcohol oxidation via increases of alcohol dehyderogenase $(A D H)$ and acetaldehyde dehydrogenase (ALDH) activities and gene expressions. Food Chemical Toxicology 50: 2508-2514.

25. Yoo, Y., E. Jung, H. Kang, I. Choi, K. Choi, and E. Jeung. 2011. The sap of Acer okamotoanum decreases serum alcohol levels after acute ethanol ingestion in rats. Int J Mol Med 28: 489-495.

26. Yu, J., H. Zhu, M. Kindy, and S. Taheri: Cytochrome P450 CYP2E1 Suppression Ameliorates Cerebral Ischemia Reperfusion Injury. Antioxidants 2021, 10. 
27. Hsu, M., S. Koike, A. Mello, L. Nagy, and F. Haj. 2020. Hepatic protein-tyrosine phosphatase 1B disruption and pharmacological inhibition attenuate ethanol-induced oxidative stress and ameliorate alcoholic liver disease in mice. Redox biology 36: 101658.

28. Chuang, C., C. Tsai, E. Lin, C. Huang, Y. Lin, C. Lan, and C. Huang: Heat-Killed Lactobacillus salivarius and Lactobacillus johnsonii Reduce Liver Injury Induced by Alcohol In Vitro and In Vivo. Molecules 2016, 21.

29. Robinson, K. E., and V. H. Shah. 2020. Pathogenesis and pathways: nonalcoholic fatty liver disease \& alcoholic liver disease. Translational Gastroenterology \& Hepatology 5: 49-49.

30. Xu, L., B. Zhao, S. Sun, S. Yu, Y. Wang, R. Ji, Z. Yang, L. Ma, Y. Yao, and Y. Chen, et al. 2020. High-dose vitamin C alleviates pancreatic injury via the NRF2/NQ01/HO-1 pathway in a rat model of severe acute pancreatitis. Annals of translational medicine 8: 852.

31. Wang, K., X. Zheng, Z. Pan, W. Yao, X. Gao, X. Wang, and X. Ding. 2020. Icariin Prevents Extracellular Matrix Accumulation and Ameliorates Experimental Diabetic Kidney Disease by Inhibiting Oxidative Stress via GPER Mediated p62-Dependent Keap1 Degradation and Nrf2 Activation. Frontiers in cell developmental biology 8: 559.

32. Dkhil, M., A. Abdel Moneim, T. Hafez, M. Mubaraki, W. Mohamed, F. Thagfan, and S. Al-Quraishy: Myristica fragrans Kernels Prevent Paracetamol-Induced Hepatotoxicity by Inducing Anti-Apoptotic Genes and Nrf2/HO-1 Pathway. Int J Mol Sci 2019, 20.

33. Zhuang, L., W. Ding, Q. Zhang, W. Ding, X. Xu, X. Yu, and D. Xi. 2021. TGR5 Attenuated Liver Ischemia-Reperfusion Injury by Activating the Keap1-Nrf2 Signaling Pathway in Mice. Inflammation 44: 859-872.

34. Shi, X., X. Jiang, B. Yuan, T. Liu, Y. Tang, Y. Che, Y. Shi, and Q. Ai. 2019. LINC01093Upregulation Protects against Alcoholic Hepatitis through Inhibition of NF-kB Signaling Pathway. Molecular Therapy-Nucleic Acids 17: $791-803$.

35. Sun, X., X. Huang, X. Zhu, L. Liu, S. Mo, H. Wang, X. Wei, S. Lu, F. Bai, and D. Wang, et al. 2019. HBOA ameliorates CCl-incuded liver fibrosis through inhibiting TGF- $\beta 1 /$ Smads, NF-KB and ERK signaling pathways. Biomed Pharmacother 115: 108901.

36. Zhou, C., H. Jia, Y. Liu, M. Yu, X. Chang, Y. Ba, and Z. Zou. 2016. Metabolism of glycerophospholipid, bile acid and retinol is correlated with the early outcomes of autoimmune hepatitis. Molecular bioSystems 12: 1574-1585.

37. Miura, H., H. Mizuguchi, M. Amano-Iwashita, R. Maeda-Kogure, A. Negishi, A. Sakai, T. Toyama, H. Kawai, A. Mitsumoto, and N. Kudo. 2021. Clofibric acid increases molecular species of phosphatidylethanolamine containing arachidonic acid for biogenesis of peroxisomal membranes in peroxisome proliferation in the liver. Biochimica et biophysica acta Molecular and cell biology of lipids 1866: 158963.

38. Hänninen, S., P. Somerharju, and M. Hermansson. 2017. Metabolic Heavy Isotope Labeling to Study Glycerophospholipid Homeostasis of Cultured Cells. Bio-protocol 7: e2268.

39. Lenoir, G., J. D'Ambrosio, T. Dieudonné, and A. Čopič. 2021. Transport Pathways That Contribute to the Cellular Distribution of Phosphatidylserine. Frontiers in cell and developmental biology 9: 737907.

\section{Figures}


AA control:

Model control: $\quad 30 \% 5 \mathrm{mg} / \mathrm{kg}$ alcohol AA treatment: $\quad 30 \% 5 \mathrm{mg} / \mathrm{kg}$ alcohol Silibinin treatment: $30 \% 5 \mathrm{mg} / \mathrm{kg}$ alcohol

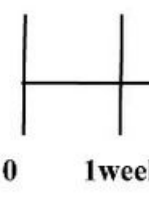

Acclimation

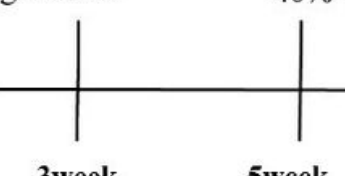

3week

$35 \% 6 \mathrm{mg} / \mathrm{kg}$ alcohol $35 \% 6 \mathrm{mg} / \mathrm{kg}$ alcohol $35 \% 6 \mathrm{mg} / \mathrm{kg}$ alcohol
$40 \% 7 \mathrm{mg} / \mathrm{kg}$ alcohol $40 \% 7 \mathrm{mg} / \mathrm{kg}$ alcohol $40 \% 7 \mathrm{mg} / \mathrm{kg}$ alcohol
$40 \% 9 \mathrm{mg} / \mathrm{kg}$ alcohol $40 \% 9 \mathrm{mg} / \mathrm{kg}$ alcohol $40 \% 9 \mathrm{mg} / \mathrm{kg}$ alcohol

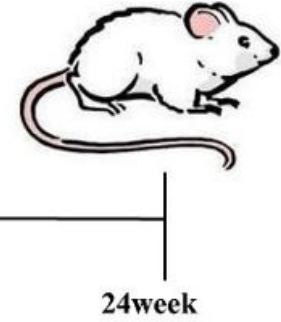

$8 \mathrm{mg} / \mathrm{kg}$ AA

$40 \% 10 \mathrm{mg} / \mathrm{kg}$ alcohol

$40 \% 10 \mathrm{mg} / \mathrm{kg}$ alcohol $+\mathrm{AA}$

$40 \% 10 \mathrm{mg} / \mathrm{kg}$ alcohol $10 \mathrm{mg} / \mathrm{kg}+$ Silibinin

\section{Figure 1}

The experimental schedule.

a

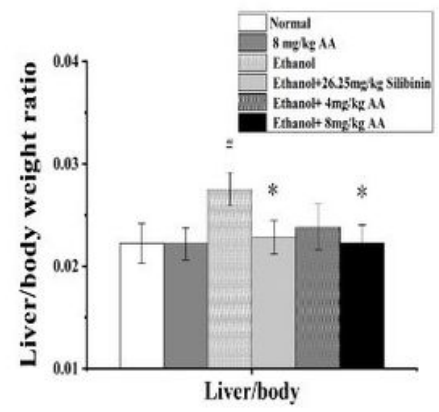

b

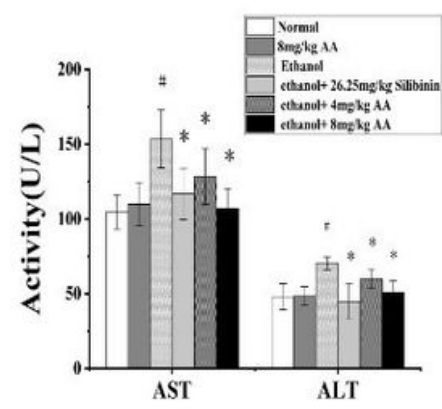

$\mathrm{C}$

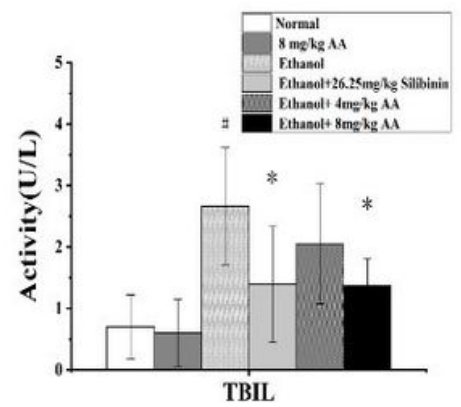

d

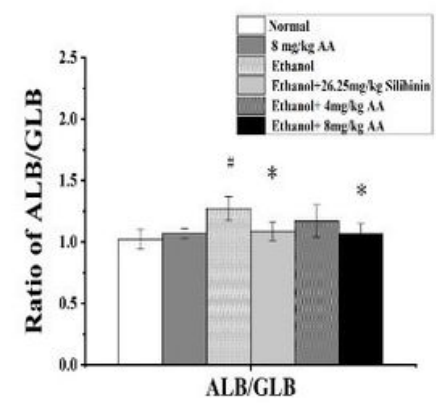

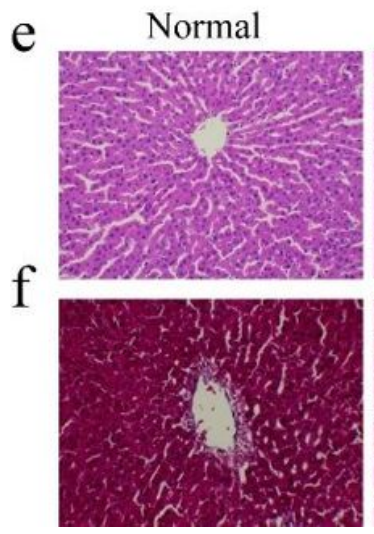

$8 \mathrm{mg} / \mathrm{kgAA}$
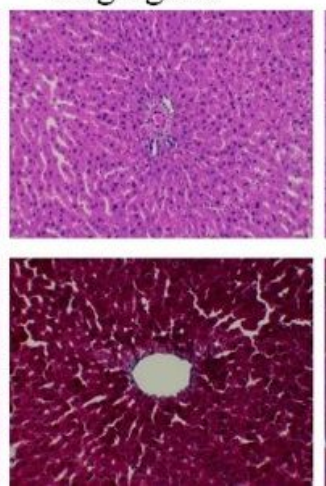

Ethanol
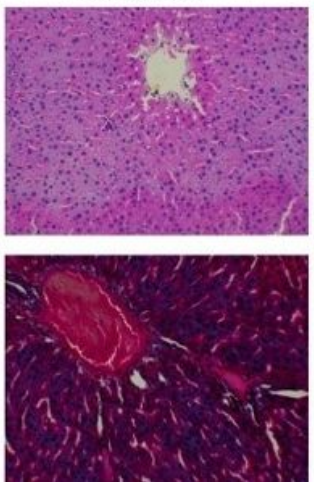

Ethanol+Silibinin Ethanol $+4 \mathrm{mg} / \mathrm{kgAA}$ Ethanol $+8 \mathrm{mg} / \mathrm{kgAA}$

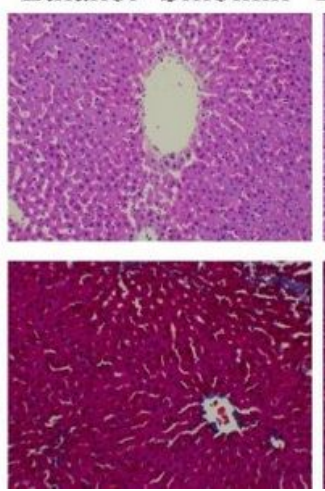

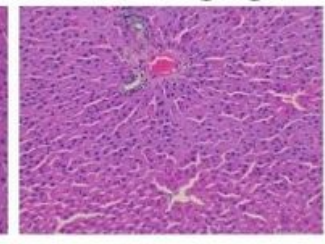
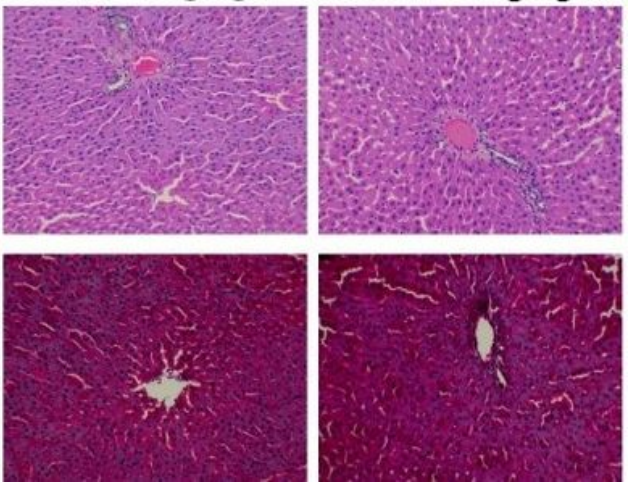

Figure 2

AA treatment ameliorated alcohol-induced abnormal liver function. (a) Liver/body weight ratio of rats. (b to d) The serum activities of ALT, AST, TBIL, and ALB/GLB were measured using an automatic biochemistry analyzer. The data are presented as mean $\pm \mathrm{SD}$. ${ }^{*} \mathrm{P}<0.05 \mathrm{VS}$. the normal group; $\# \mathrm{P}<0.05 \mathrm{VS}$. the model group. (e) Hepatic histological changes were observed by H\&E staining (200x); (f) Collagen accumulation conditions were observed by Masson's trichrome staining (200x). 
a

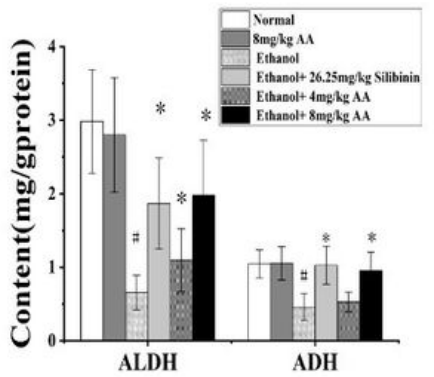

d

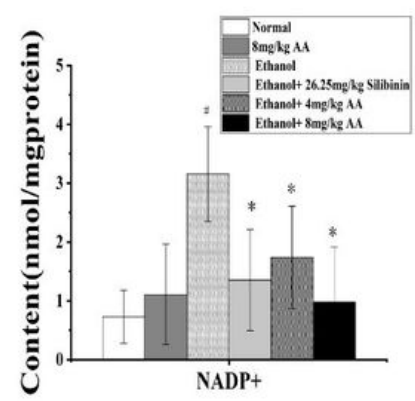

g

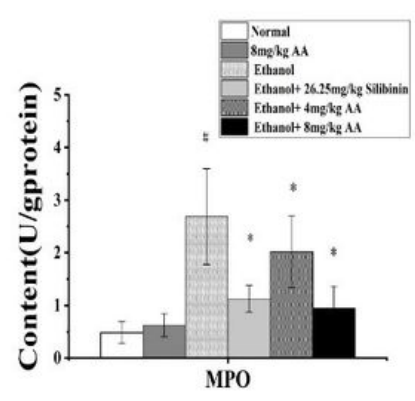

j

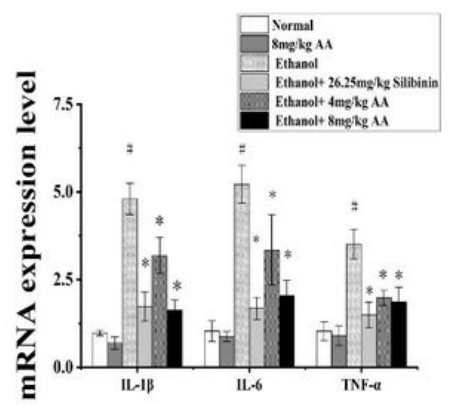

b

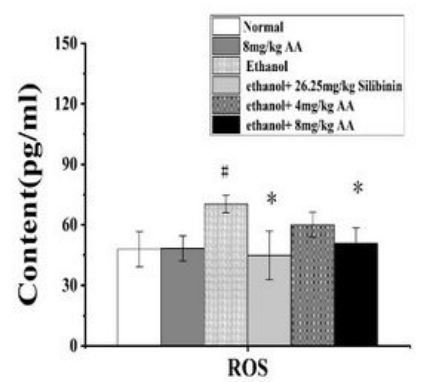

$\mathrm{e}$

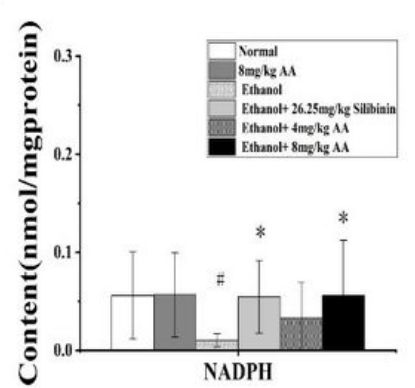

h

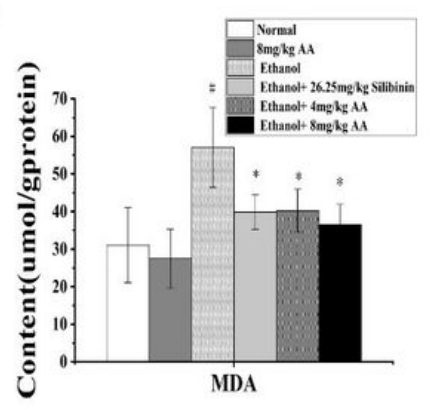

c

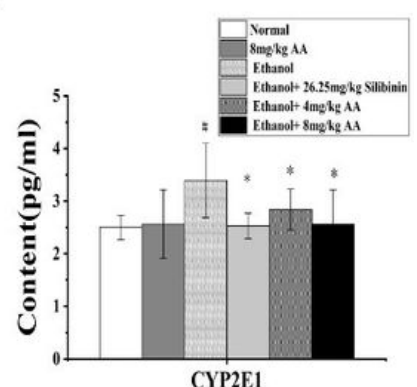

f

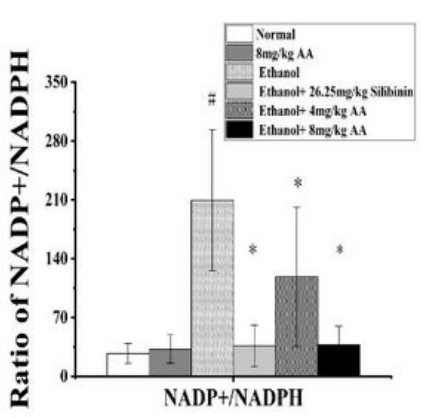

i

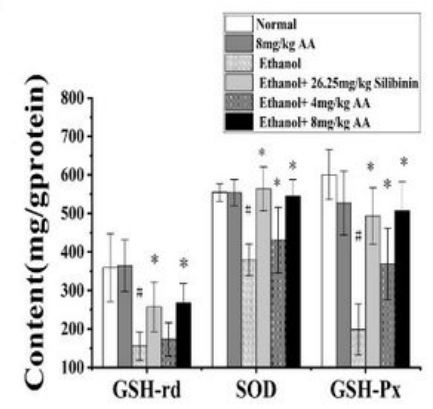

Figure 3

AA treatment alleviated metabolic enzyme activity and oxidative stress, as well as suppressed pro-inflammatory cytokines. (a) The content of ADH and ALDH were detected by commercially available kits. (b and c) An enzyme-linked immunosorbent assay was used to detect the content of ROS and CYP2E1. ( $d$ to $f$ ) The content of NADPH, NADP+ and the ratio of NADP+/ NADPH were detected by commercially available kits. ( $\mathrm{g}$ to i) The content of MPO, MDA, GSH-rd, SOD, and GSH-px was detected by commercially available kits. (j) The mRNA levels of TNF-a, IL6, and IL-1 $\beta$ were detected by the qPCR assay. The data are presented as mean \pm SD. ${ }^{*} P<0.05 \mathrm{VS}$. the normal group; $\# P<0.05 \mathrm{VS}$. the model group. 


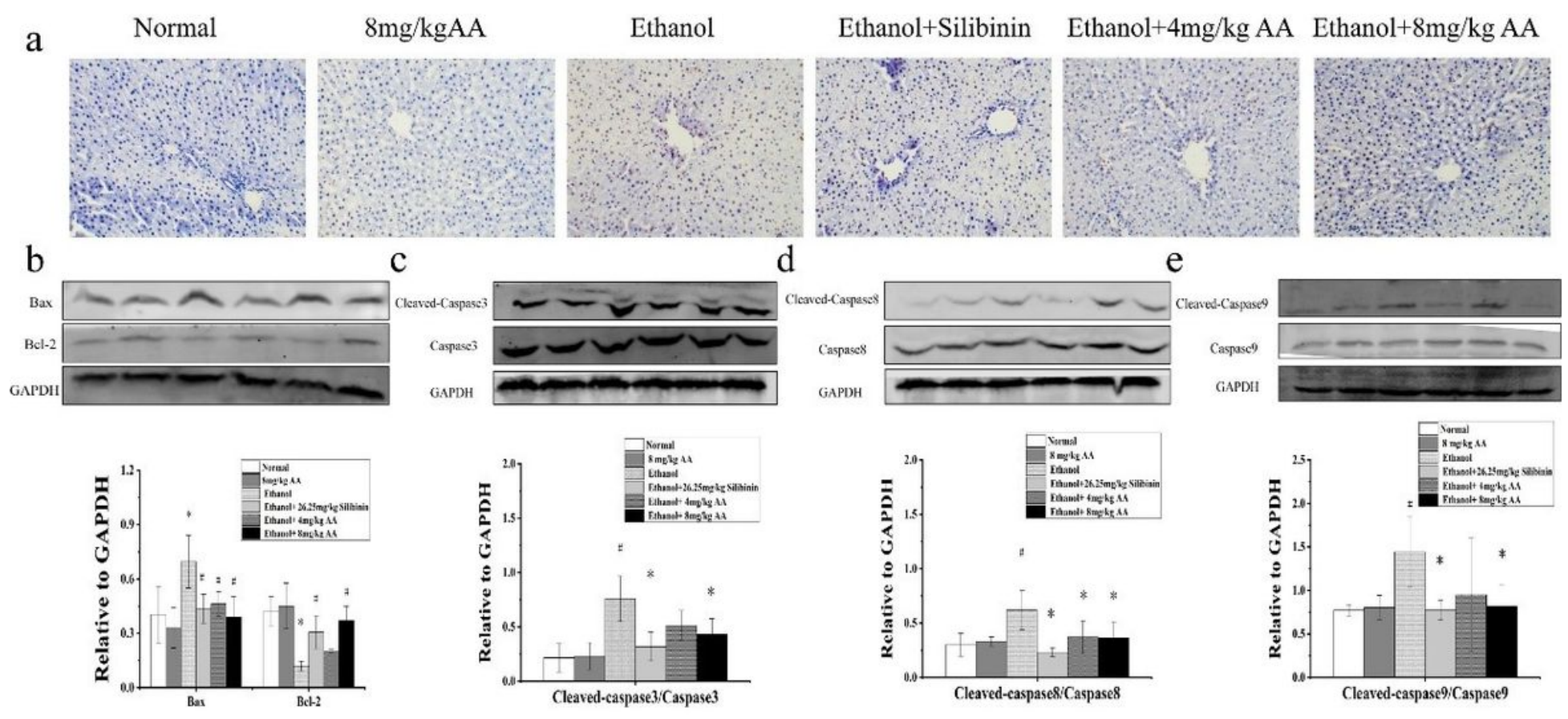

Figure 4

AA treatment alleviated alcohol-induced hepatocyte apoptosis. (a) Hepatocyte apoptosis was assessed by TUNEL staining (200x); (b) The protein levels of Bax and Bcl-2 were determined by western blot; (c to e) The protein levels of Cleaved caspase 3, caspase 3, Cleaved caspase 8, caspase 8, Cleaved caspase 9, and caspase 9 were determined by western blot. The bands 1 to 6 represent the normal control, AA control, Model, Silibinin-treated group, low- and highdosages of AA treated groups, respectively. The data are presented as mean $\pm \mathrm{SD}$. ${ }^{*} \mathrm{P}<0.05 \mathrm{VS}$. the normal group; \#P< 0.05 VS. the model group. 

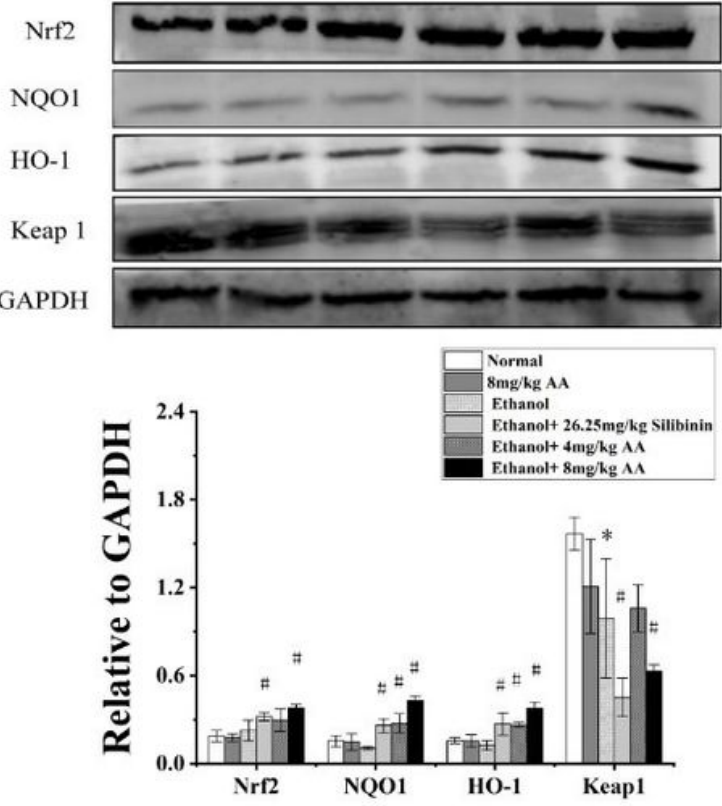

$\mathrm{c}$

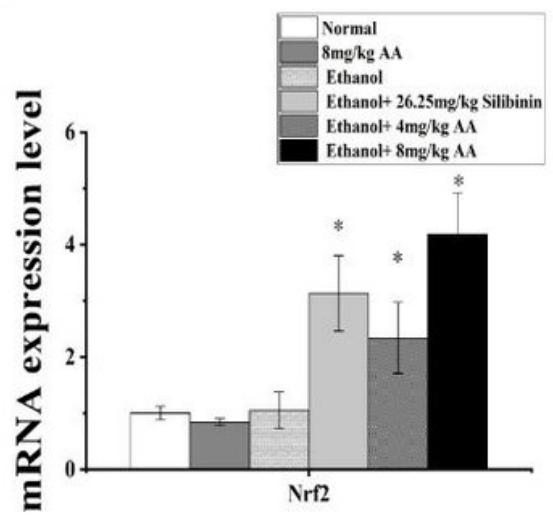

b
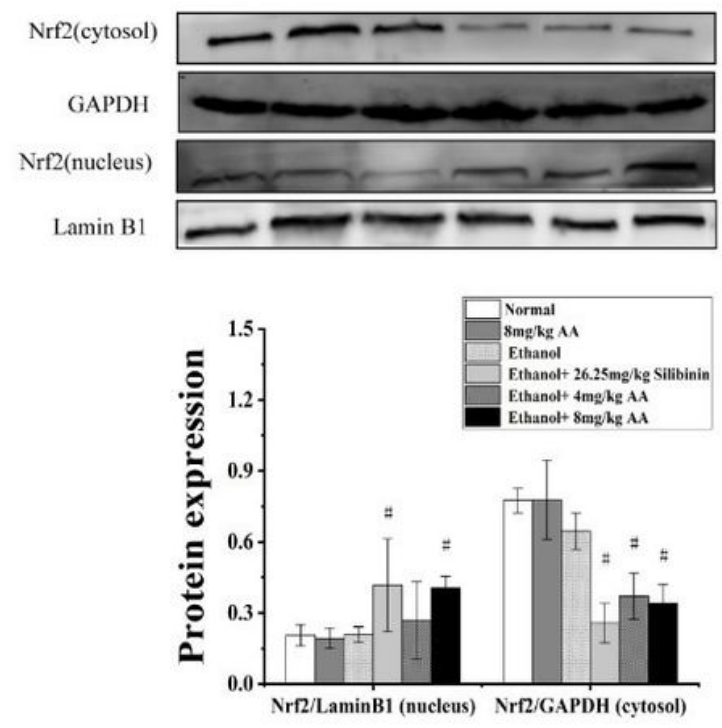

$\mathrm{e}$

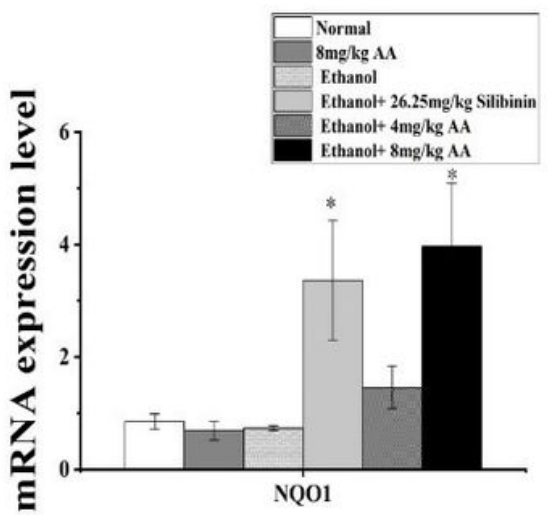

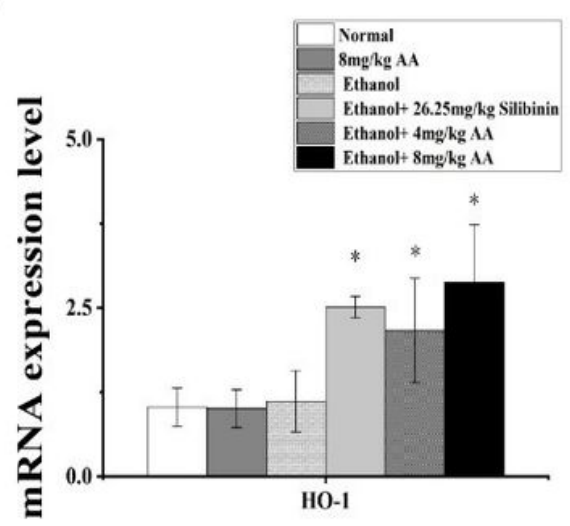

Figure 5

AA treatment activated hepatic the Nrf2 signaling pathway. (a) The protein levels of Nrf2, NQO1, HO-1, and Keap 1 were determined by western blot. (b) The cytosol and nucleus protein expressions of Nrf2 were determined by western blot. (c to e) The mRNA levels of Nrf2, NQ01and HO-1 were detected by the qPCR assay. The bands 1 to 6 represent the normal control, AA control, Model, Silibinin, low- and high-dosages of AA treated groups, respectively. The data are presented as mean $\pm S D$. ${ }^{*} P<0.05$ VS. the normal group; $\# P<0.05$ VS. the model group. 
a
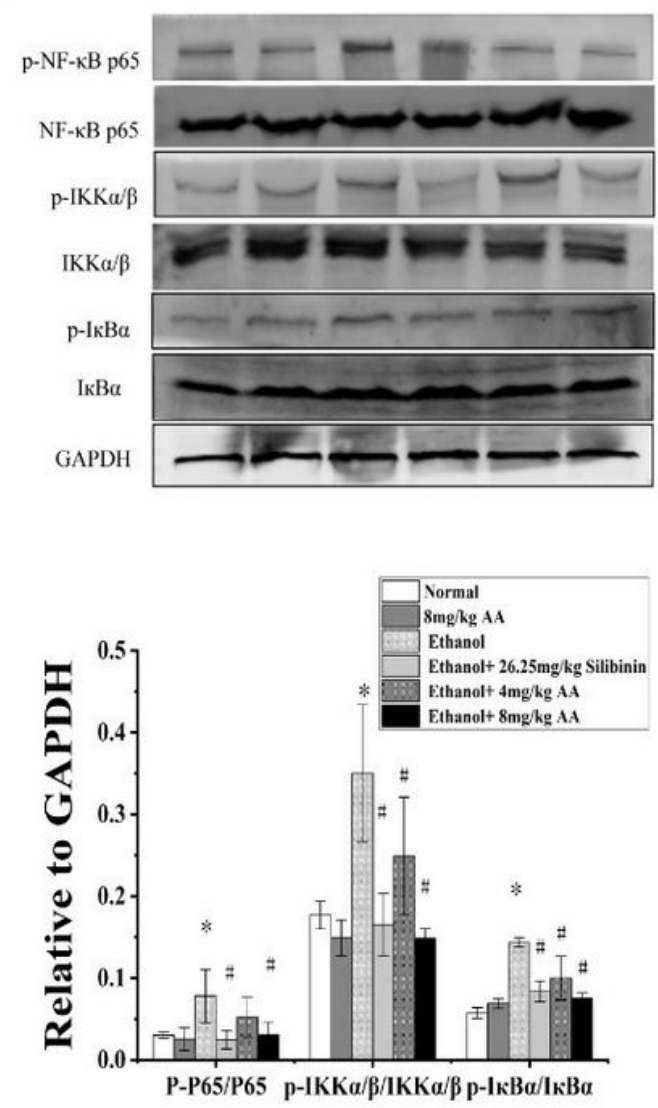

b
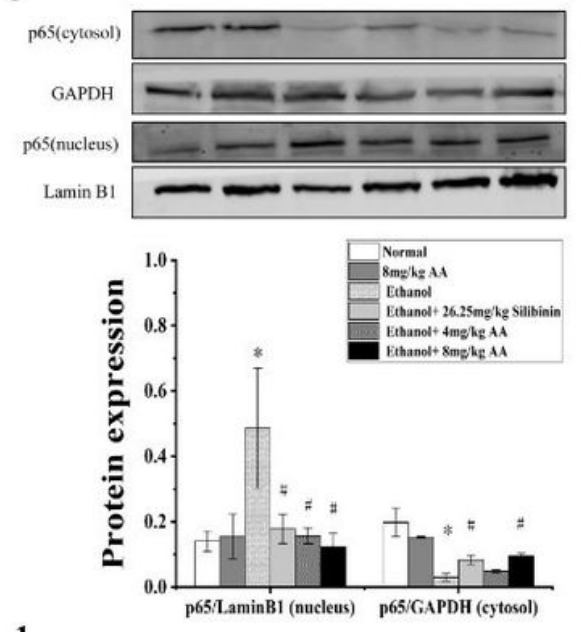

d

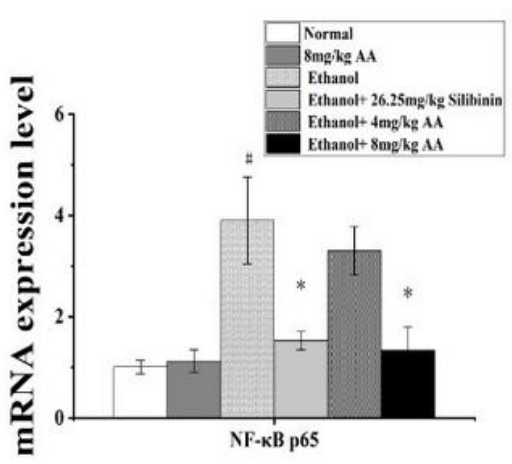

c
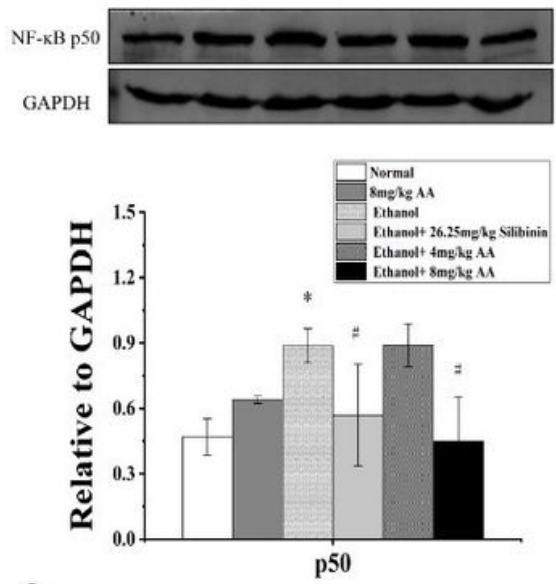

e

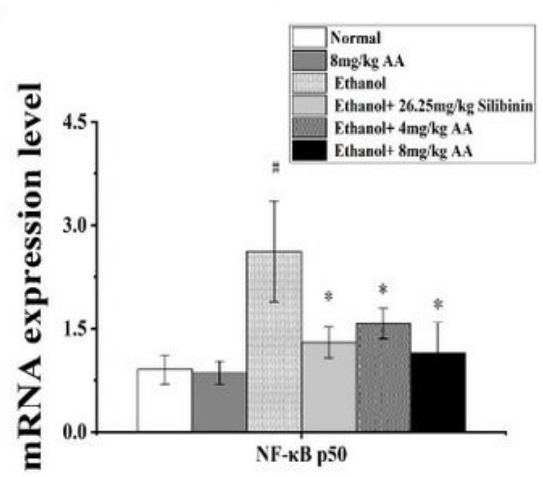

Figure 6

AA treatment inhibited hepatic NF-KB activation. (a) The protein levels of the phosphorylation of NF-KB p65, IKKa/ $\beta$, and IKBa were determined by western blot. (b) The cytosol and nucleus protein expressions of NF-KB p65 were determined by western blot. (c) The protein level of NF-kB p50 was determined by western blot. The bands 1 to 6 represent the normal control group, AA control, Model, Silibinin, low- and high-dosages of AA treated groups, respectively. (d and e) The mRNA levels of NF-KB p65 and NF-KB p50 were detected by the qPCR assay. The data are presented as mean \pm SD. ${ }^{*} \mathrm{P}<0.05 \mathrm{VS}$. the normal group; $\# \mathrm{P}<0.05 \mathrm{VS}$. the model group. 
a

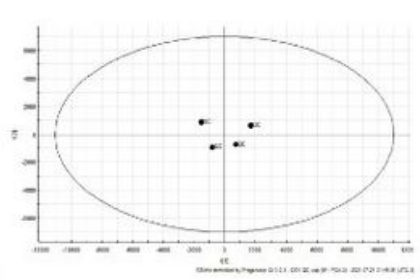

e

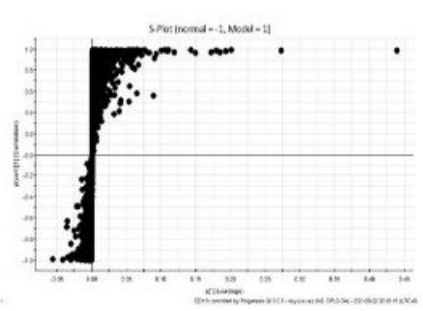

$\mathrm{h}$

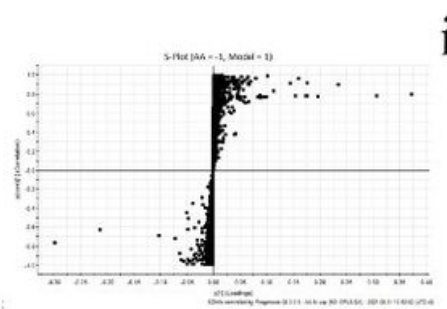

b

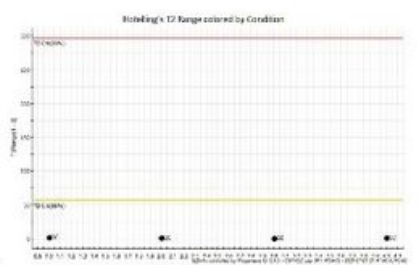

f
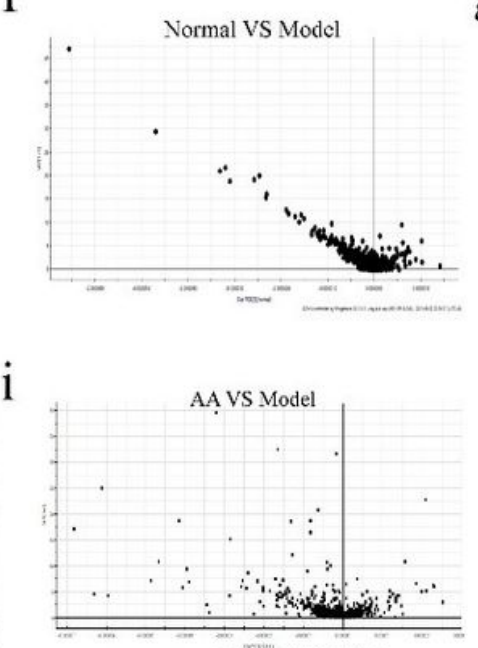

c

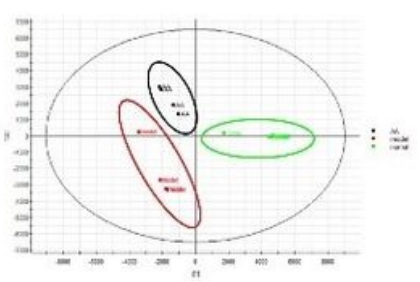

$\mathrm{g}$
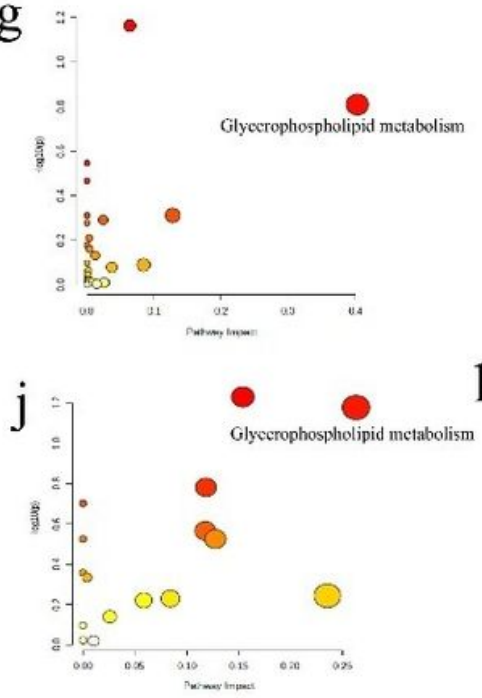

d

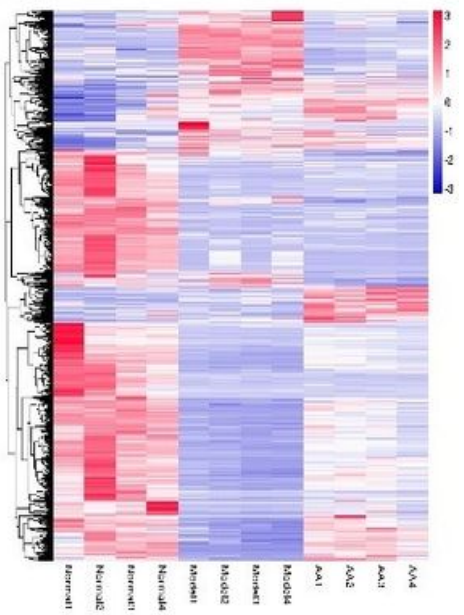

$\mathrm{k}$

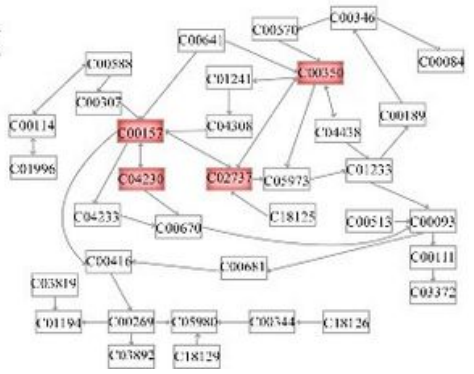

Figure 7

Metabolome analysis of the normal, AA, and model group. (a) Principal Component Analysis (PCA) diagram of QC; (b) Hotelling's T2 Range plot; (c) PCA diagram; (d) Volcano plot; (e) S-Plot diagram for normal VS model group; (f)Variable importance plot (VIP) for normal VS model group; (g) Metabolic pathways enrichment for the normal and model group (each point represents a metabolic pathway; the size of dot means the impact; the color (red) represents the positive correlation). (h) S-Plot diagram for AA VS model group; (i)Variable importance plot (VIP) for AA VS model group; (j) Metabolic pathways enrichment for the AA and model group (each point represents a metabolic pathway; the size of dot means the impact; the color (red) represents the positive correlation); (k) Glycerophospholipid metabolism pathway. Metabolites in red (C00350, C00157, C04230, and C02737) were the potential compounds regulated by the AA. 
a

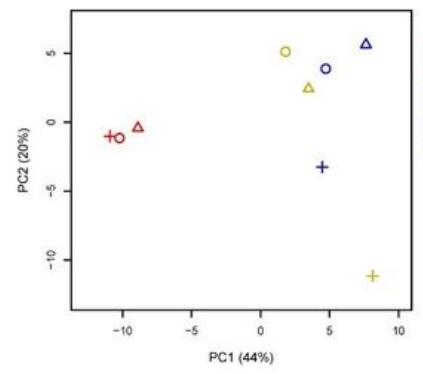

C

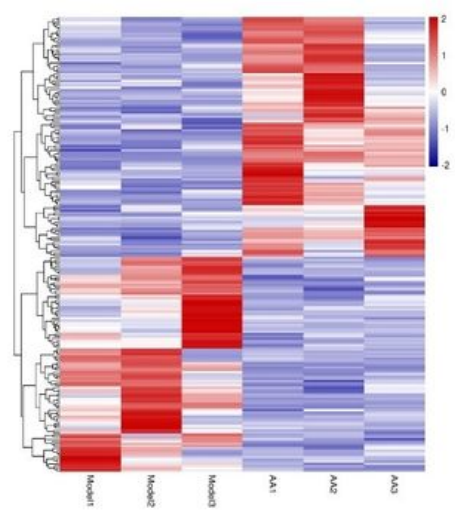

b

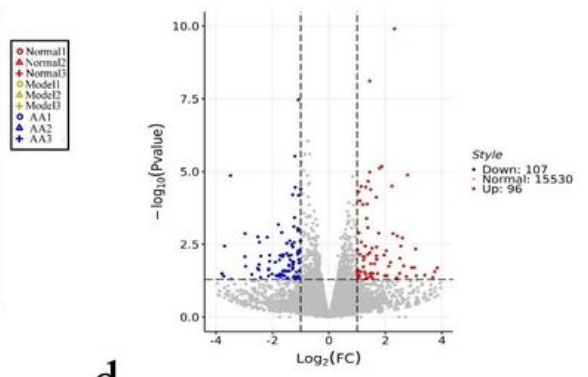

$\mathrm{d}$

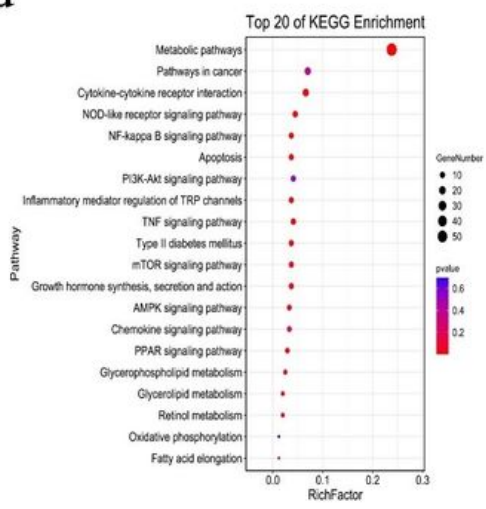

e

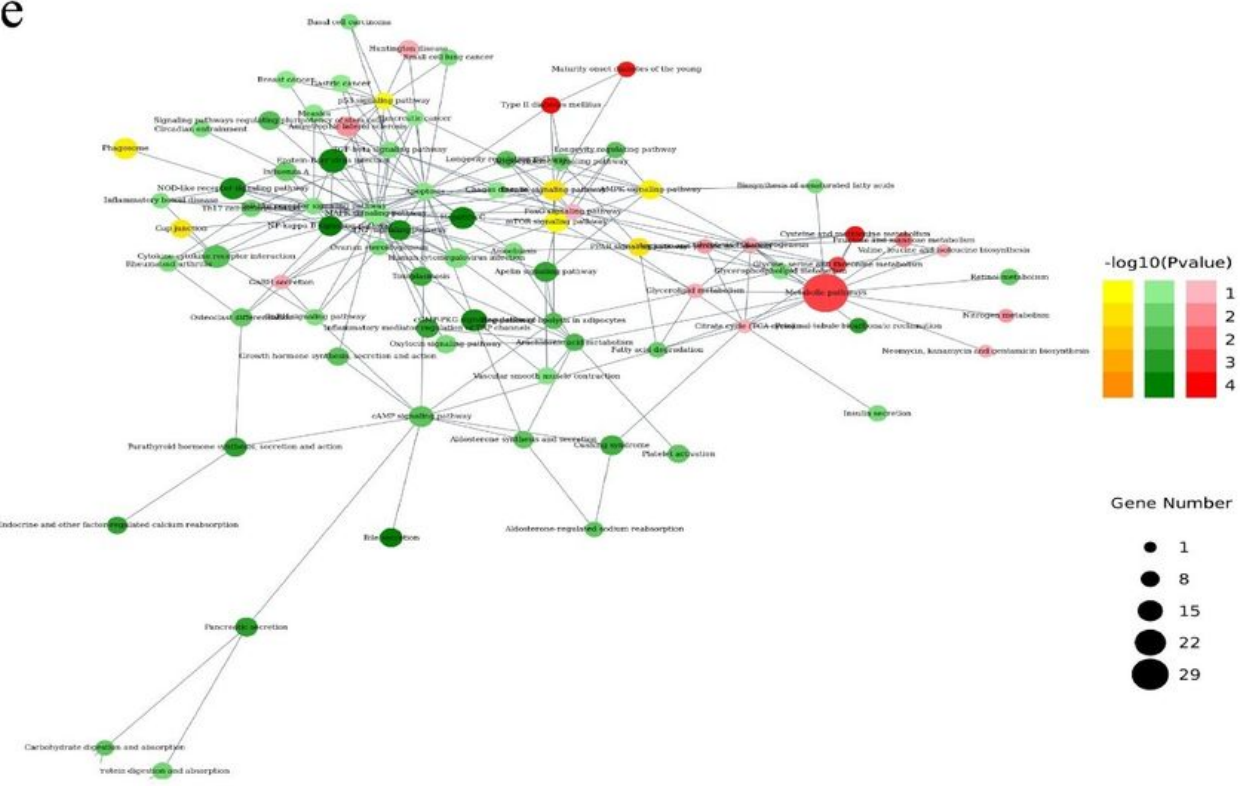

Figure 8

Transcriptomics analysis between the AA and model group, and integrated analysis of metabolomics and transcriptome. (a) PCA plot; (b) Heatmap; (c) Volcano plot; (d) KEGG pathway analysis; (e) Network was built based on the relationship of KEGG Pathways. 
a

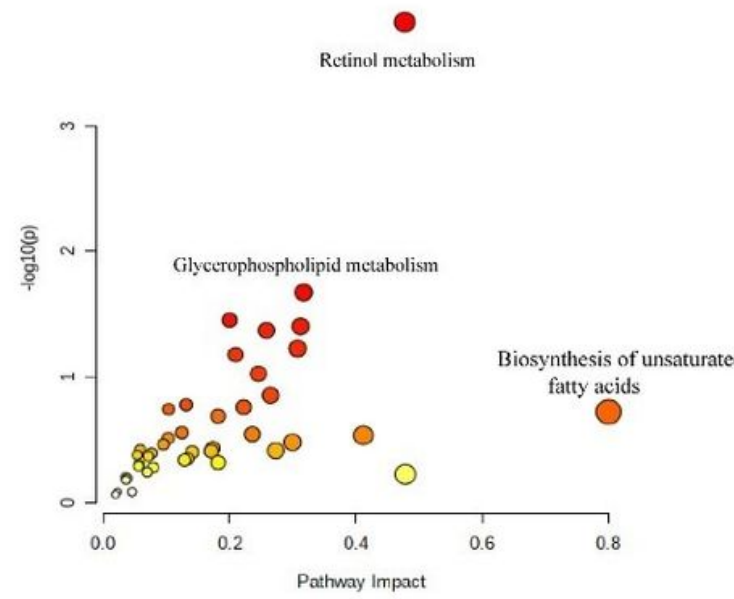

$\mathrm{C}$

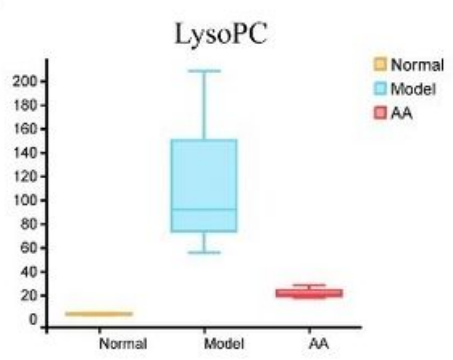

PC b

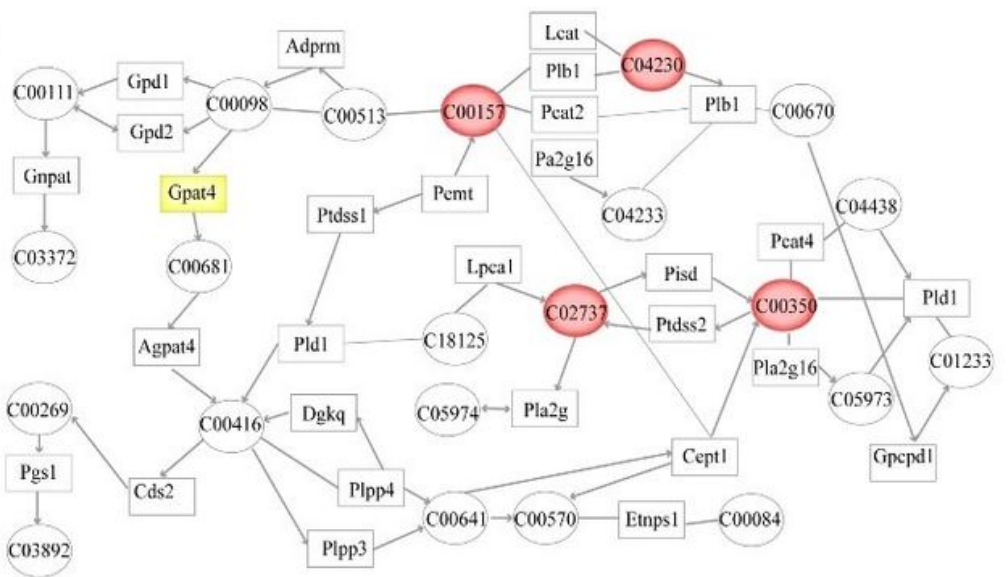

$\mathrm{e}$

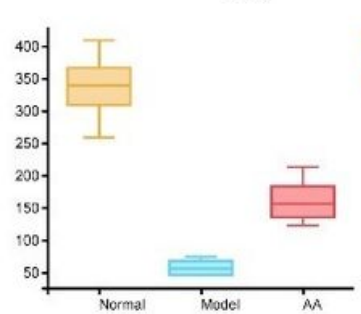

f

PE

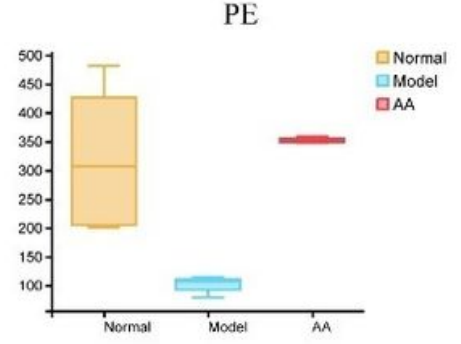

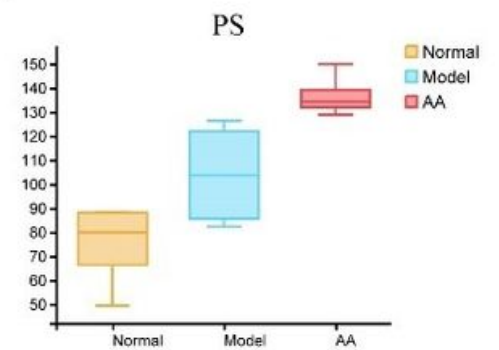

Figure 9

Integrated analysis of metabolomics and transcriptome. (a) Metabolic pathways enrichment (each point represents a metabolic pathway; the size of dot means the impact; the color (red) represents the positive correlation); (b) Glycerophospholipid metabolism pathway. Metabolites in red (C00350, C00157, C02737, and C04230) and genes in yellow were the potential targets regulated by the AA; (c to f) Metabolites' expression of LysoPC, PC, PE, and PS. 


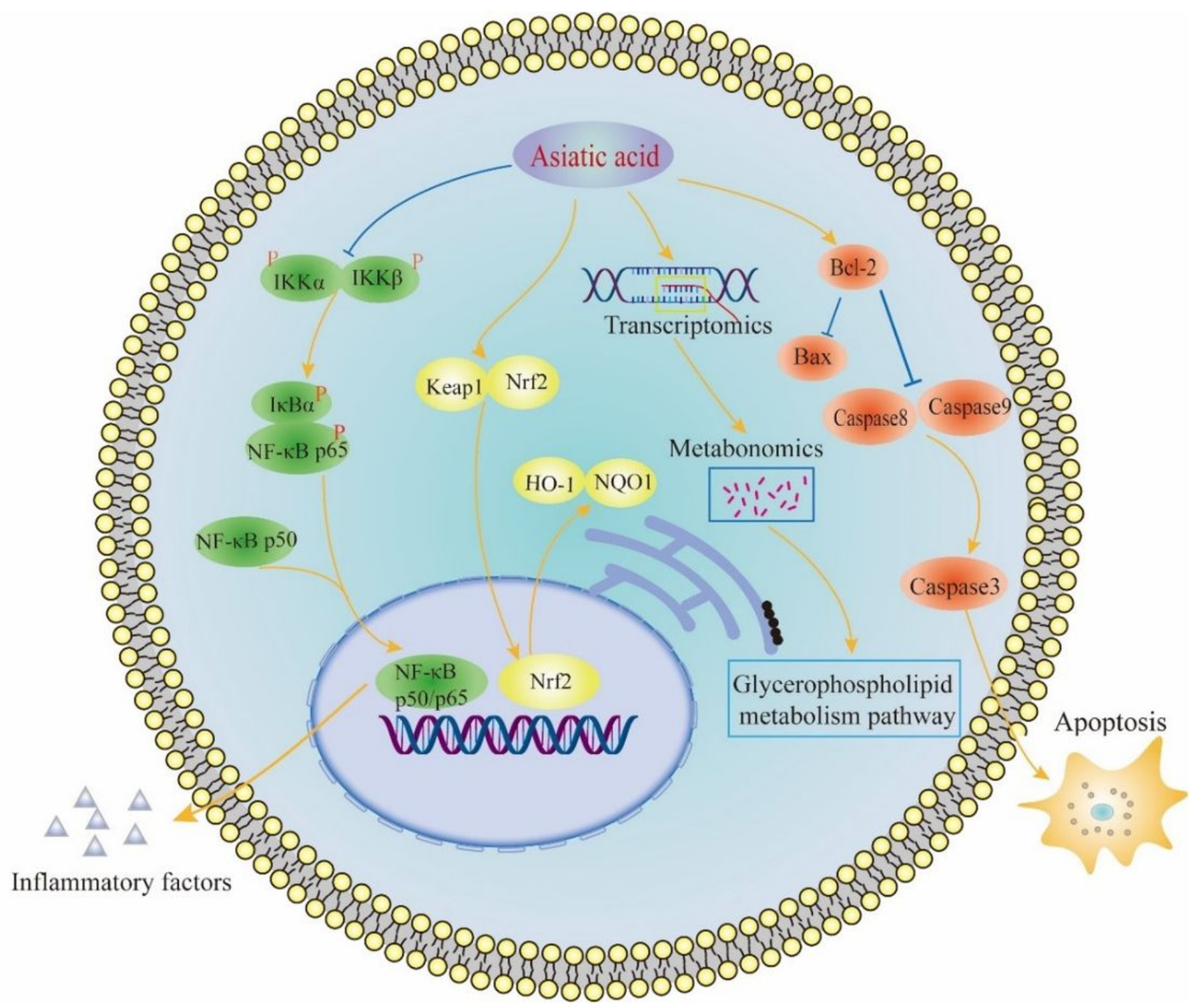

Figure 10

AA activated the Nrf2 and inhibited NF-KB signaling pathway, and promoted Glycerophospholipid metabolism, ultimately alleviating hepatocyte inflammation, apoptosis, and damage in rats with $\mathrm{AH}$. 